\title{
Multi-depot home health care routing and scheduling problem with multimodal transportation: Mathematical model and solution methods
}

\author{
Fahimeh Ghiasvand Ghiasi, Mehdi Yazdani ${ }^{*}$, Behnam Vahdani, Abolfazl Kazemi \\ Department of Industrial Engineering, Faculty of Industrial and Mechanical Engineering, Qazvin Branch, \\ Islamic Azad University, Qazvin, Iran
}

\begin{abstract}
Providing appropriate home health care is one of the increasing concerns in the health care organizations. Home Health Care provides various services for disabled or elderly individuals at their homes. Also, deal with the current critical situation of the coronavirus disease (COVID-19) due to the limited capacity of hospitals and the feeling of insecurity in crowded places, home health care is more recommended. This paper addresses a Home Health Care Routing and Scheduling Problem (HHCRSP) with two modes of transportations including public and private modes. Also, multi-depot version of the problem is studied to enhance the service delivery in scattered points. In this study, a mathematical model is presented based on a Mixed Integer Linear Programming (MILP) whose objective function is minimization of the sum of the travel distance and overtime costs. Furthermore, three meta-heuristic algorithms including Invasive Weed Optimization (IWO), Grasshopper Optimization Algorithm (GOA) and Simulated Annealing (SA) are presented for solving the large-sized problems. Since the performance of meta-heuristic algorithms depends on setting the parameters, Taguchi method is used to statistically set parameters of the developed algorithms. The computational results have shown that the proposed IWO has worked better than the other two proposed algorithms statistically.
\end{abstract}

Keywords: Home Health Care; Multiple Depots; Multimodal Transportation; Routing; Scheduling; Mixed Integer Linear Programming; Meta-heuristic

\footnotetext{
*. Corresponding author. E-mail address: mehdi_yazdani2007@yahoo.com, Tel.: +989113310090 (M. Yazdani); f.gh.ghiasi@gmail.com (F. Ghiasvand Ghiasi); b.vahdani@gmail.com (B. Vahdani); abkaazemi@qiau.ac.ir (A. Kazemi)
} 


\section{Introduction}

Home Health Care (HHC) services present specialized and general medical care for elderly and disabled ones who need continuous nursing care at their homes. Currently, a vast number of medical and nursing companies are working in this field [1,2]. Uncontrolled growth of industrial pollution as well as urban transportation system, have led to a raise in hospitalization. Although the hospital offers specialized services, the congestion of hospitals is inconsistent with providing high quality services. The lower patients be in the hospital, the better nurses will be able to take care of them. On the other hand, patients who need general hygiene cares feel more comfortable at home [3]. Also, with the advent of the coronavirus it's better for high risk people or patients with mild symptoms of this disease to get required medical services at home. Moreover, in cases where COVID-19 patients are in recovery phase some patients can leave the hospital and continue treatment at home.

Since the HHC goes back to public welfare and health, there are many important challenging dilemmas in this area such as Home Health Care Routing and Scheduling Problem (HHCRSP). Urbanization made nursing companies more eager to find the best route and schedule for nurses systematically. The HHCRSP is defined as planning and directing nurses to provide cares or services at patients' homes. It is developed form of Vehicle Routing Problem (VRP) with time windows. To the best of our knowledge, Fernandez et al. [4] are one of the first researchers that have studied the HHCRSP. They examined the working day of the United Kingdom nurses and considered the travel time and service providing time in the presented model. Also, the authors provided a method to split up the country based on the allocation of nurses. Begur et al. [5] has addressed a Spatial Decision Support System to plan HHC medical services.

The HHCRSP can be divided into two categories: Single depot VRP $[6,7,8]$ and multiple depots VRP $[1,9,10]$. In single depot mode, nurses departure from a health center to patients' home. Braekers et al. [6] developed the multi-objective single depot version of HHCRSP. They proposed a Mixed Integer Linear Programming (MILP) model for this problem and examined the tradeoff between cost and customer dissatisfaction objectives. The model considered characteristics such as overtime [11,12], travel costs depending on the mode of transportation, hard time window and client preferences. In their study, small sizes of problems were solved using epsilon constraint framework. Also, the meta-heuristic algorithm based on the multi-directional local search framework and large neighborhood search was proposed to solve problem instances of realistic size. In order to assessing urban transportation sustainability, Fikar and Hirsch [7] considered car and trip sharing concepts for the single depot HHCRSP. They also have enumerated walking and trip sharing as an effective way to attain sustainability goals. In multiple depots subject, there are two approaches in the literature. In the first approach, each nurse's home acts as a depot and in the second approach, nurses move from several HHC offices to patients' homes and the destination depot will be at a final location, e.g. their home, office, hospital or laboratory [10]. Note that far too little attention has been paid to second approach of multiple depots. Decerle et al. [1] developed a mixed integer programming model for the multi-depot HHC. Each nurse had a hard time window 
and a special qualification level. Their planning horizon was daily and a meta-heuristic algorithm was presented for the problem. Tohidifard et al. [10] addressed a HHCRSP with several depots and time windows. They proposed a bi-objective mathematical model and four heuristics for Green Home Health Care (GHHC) problem. GHHC was developed to get HHC closer to real world. Also, different types of transportation system were used in the research problem. Nowadays there are an increasing demand for HHC services, so the world is facing with the growth of HHC service delivery organizations. Also, HHC organizations are developing their branches in cities and metropolises. Often, the main HHC center is a hospital with full facilities. In addition to this hospital, some branches are stablished in different high demanded places to present services in a scattered manner. The task of the branches is to equip nurses with necessary items and direct them to the patients for doing needed services. So, we chose multiple depots mode to present and define the problem in this article.

Most articles in the HHC field have used single transportation mode and a few of them have used multimodal transportation [13]. VRP literature tried to control environmental pollutants using approaches such as utilizing several vehicle types [9], different modes of transportation and car sharing [7]. Xiao and Konak [14] developed a MILP model with considering heterogeneous vehicles. In their research different types of transportation system have been used. These vehicle types have different capacities and cost of transportation. In the present article, we focused on two modes of transportation including public and private modes [15, 16] with homogeneous vehicle types. Since most of researches model the HHC as a development of VRP, the travel distance cost is considered as the primary objective function of the problem. Moreover, several studies of HHC include overtime [17] in their models.

Even if all of three above mentioned subjects including overtime, multimodal transportation and multiple depots are investigated in the HHC literature, few articles addressed all of them simultaneously. In this study, a mathematical model is presented for the HHCRSP problem based on a MILP whose objective function is minimization of the sum of the travel distance and overtime costs. Also, second approach of multiple depots and multimodal transportation (public and private modes) are considered in developing this model. Therefore, this article considers overtime, multimodal transportation and multiple depots subjects at the same time in the presented problem.

A review of the literature reveals that some authors provided exact methods for solving the HHCRSP. Liu et al.[18] presented a branch-and-price algorithm for the problem. Riazi et al. [19] developed a column generation-based gossip algorithm for a single depot HHCRSP which contains single mode of transportation. Authors integrated the gossip algorithm with a local solver based on column generation, which made it an effective algorithm for larger problem instances. The objective function of the problem was determined minimization of the total distance traveled by all of the caregivers. Despite the benefits of accurate methods, these methods are able to plan a few visits and a limited number of nurses per day. Since the HHCRSP belongs to NP-hard class [12], exact methods cannot tackle this problem within a reasonable amount of time. To overcome this difficulty, most of studies have utilized meta-heuristic algorithms to solve the problem in the 
HHC literature. These meta-heuristics include population-based methods such as hybrid ant colony algorithm [20], variable neighborhood search [17] and Genetic Algorithm [21]. Hiermann et al. [12] generated the initial response by a random approach, then improved the quality of solution with four meta-heuristics. Also, Decerle et al. [22] developed the memetic algorithm for solving the HHCRSP and investigated impact analysis of workload balancing on the problem. Trautsamwieser et al. [17] solved the real size HHCRSP problems by heuristic-based variable neighborhood search. Also, Mankowska et al. [23] developed adaptive variable neighborhood search meta-heuristic for the HHCRSP with interdependent services. Liu et al. [24] proposed an adaptive large neighborhood search heuristic for the vehicle routing problem with time windows of nurses and patients and synchronized visits in the HHC industry. The problem objective function was determined minimization of the vehicles' travel costs and fixed costs. In the present study, we propounded three well-known meta-heuristics including Invasive Weed Optimization (IWO), Simulated Annealing (SA) and Grasshopper Optimization Algorithm (GOA) for solving the understudied problem. To the best of our knowledge, it is the first time that IWO and GOA metaheuristic algorithms are used to optimize the HHCRSP problem. The HHCRSP covers some of the problems and challenges related to nursing companies in the real world. Mainly HHCRSP encompass two aspects of routing and scheduling. Therefore, studying and optimizing HHCRSP bring about a reduction in costs of both patients and $\mathrm{HHC}$ centers, service providing in a reasonable time and also covering more patients in a day. According to the fact that the problem is NP-Hard, we tried to provide more efficient algorithms for solving this problem. The main highlights of the paper are as follows:

- Presenting a suitable and validated mathematical model for the HHCRSP based on a MILP;

- Considering overtime, multimodal transportation (public and private modes) and multiple depots subjects at the same time and simultaneously in the presented problem;

- Developing SA, IWO and GOA meta-heuristic algorithms to optimize the HHCRSP;

- Presenting a new solution representation method for proposed meta-heuristic algorithms.

The outline of the paper is as follows. In section 2, problem description is presented. Section 3 introduces the new mathematical model for the studied problem. In section 4, presented metaheuristic approaches are described, also computational study results are provided in section 5. Finally, conclusion and future perspectives of our work are stated in section 6.

\section{Problem description}

The HHCRSP is developed on a directed graph $G=(V, Q)$ where $V=\left\{V_{p} \cup V_{s} \cup V_{e}\right\}$ is the set of vertices representing patients' nodes $\left(V_{p}\right)$, initial start depots $\left(V_{s}\right)$ and destination depot $\left(V_{e}\right)$. Each nurse $n \in N\left(N=\left\{N_{1}, N_{2}, \ldots, N_{n_{\text {max }}}\right\}\right)$ has a service time $\left(s t_{i}\right)$ for patient $i$. For each nurse $n$, a time window $\left[a_{n}, b_{n}\right]$ is defined, which specifies the lower and upper bound of the nurse total activity during a day. Maximum regular working time duration for each nurse $n$ is $r_{n}$ and $m_{n}$ is 
maximum allowed daily working time duration for each nurse, $r_{n} \leq m_{n}$. Therefore, total working time is limited and the overtime is allowed up to a determined cost of $d_{n}$. Overtime is equal to the time exceeding the maximum regular working day. Each nurse is able to provide specific services and is assigned to the patients according to his/her qualification level. Parameter $q_{\text {in }}$ determines whether a nurse $n$ is sufficiently qualified to visit patient $i\left(q_{i n}=1\right)$ or not $\left(q_{i n}=0\right)$. In order to all nurses can go to initial start and destination depots, the values of $q_{0 n}$ and $q_{f n}$ is set to 1 . The arc set $Q$ is characterized as $Q=\left\{(i, j, n) \mid i \in V \backslash V_{e}, j \in V \backslash V_{s}, n \in N, i \neq j, q_{i n}=1, q_{j n}=1\right\}$. In the problem of this article, it is assumed that a qualified nurse is predetermined by the management of medical centers to provide the required medical services for each patient (job). Therefore, each nurse has a pre-selected set of patients who should only visit them, which should be considered in the members of the set $Q$. In fact, the challenge of the presented article is related to a real-world situation in which patients have selected their own nurse based on their preferences and finalized their request in a contract with the main health care center. For patient convenience a hard time window $\left[e_{i}, l_{i}\right]$ is defined for each patient $i$. Nurses should wait if they arrive before the start of time window. The nurses' movements between the nodes are done by vehicles. In this study, two types of transportation including private (mode 1) and public (mode 2) modes are considered for used vehicles. The public transportation mode has fewer travel costs and higher travel time compared with private transportation mode. $K=\left\{K_{1}, K_{2}, \ldots, K_{k_{\max }}\right\}$ is the set for vehicles and $U=\left\{u_{1}, u_{2}, \ldots, u_{k_{\max }}\right\}$ is the set for modes of transportation related to vehicles. Since in the understudied problem, each nurse is assigned to one vehicle for trip, the number of nurses is equal to the number of available vehicles $\left(n_{\max }=k_{\max }\right)$. Each vehicle $k \in K$ begins at one of the initial start depots (initial depots), transports the nurse between patients' nodes and ends at destination depot (final depot). Parameter $c_{i, j, u_{(k)}}$ is the travel cost between nodes $i$ and $j$ by vehicle $k$ under mode $u_{(k)}$ and $t_{i, j, u_{(k)}}$ travel time between nodes $i$ and $j$ by vehicle $k$ under mode $u_{(k)}$. The values of these two parameters are computed based on the Euclidean distance between nodes and coefficients of time and cost.

The developed HHC problem has a daily planning horizon. At the beginning of each day, the problem is solved according to the real situation, location of new patients and the nurse presence. Afterwards, each nurse can start their visits by assigned vehicle according to the scheduled sequence. In this study, customer is essentially a patient who needs specialized medical and nursing services at home. These services can include injections, changing a surgical wound dressing, removing stitches and other essential medical services. Nurses have multiple initial depots and one final depot. In this problem initial depots are health centers which should be assigned to nurses for determining starting point and the final depot is a hospital. It should be noted that all the health centers are organized and managed by a hospital. Nurses start their route from 
one of the health centers and finally come back to the hospital to deliver the blood sample and infectious wastes such as syringe needles. The problem goal is to find the best schedule and route for each nurse including:

1. Selecting the initial start depots for nurses;

2. Determining the sequence of the patients' visitation for each nurse;

3. Finding the mode of transportation for each nurse based on the route.

Also, the problem objective function is sum of the travel distance and overtime costs minimization.

In the following example, a problem is assumed wherein there are 6 patients, 3 nurses, 3 vehicles, 2 initial centers and one final health care center. Vehicles $K_{1}$ and $K_{3}$ are belonged to transportation mode 1 (private mode) and vehicle $K_{2}$ to transportation mode 2 (public mode). It is predetermined that nurse1 $\left(N_{1}\right)$ visits patient1 $\left(V_{p 1}\right)$ and patient3 $\left(V_{p 3}\right)$, nurse $2\left(N_{2}\right)$ visits patient2 $\left(V_{p 2}\right)$ and patient5 $\left(V_{p 5}\right)$ and nurse3 $\left(N_{3}\right)$ visits patient4 $\left(V_{p 4}\right)$ and patient6 $\left(V_{p 6}\right)$. Parameters values related to patients' service times $\left(s t_{i}\right)$, time window of nurses $\left[a_{n}, b_{n}\right]$ and time window of patients $\left[e_{i}, l_{i}\right]$, maximum regular working time $\left(r_{n}\right)$ and maximum allowed daily working time $\left(m_{n}\right)$ are shown in Table 1 . The coordinates of nodes for this problem are generated randomly and travel distance is calculated by Euclidean distance method. Then, travel time and travel cost are computed based on obtained Euclidean distance between nodes. Nurses' routes, assigned vehicles to the nurses, travel time of nurses from one node to another node and patients' service time (in terms of minutes) related to one feasible solution of this problem are shown in Figure 1. The values of travel times are dependent on the transportation modes. In this graph the nurse's route is illustrated from origin nodes to destination. The Gantt chart of this solution is shown in the Figure 2. In this chart, the vertical axis represents nurses whereas the horizontal axis represents time. The latest arrival time to final health center is 446.64 and it is belonged to nurse $N_{2}$. Also, the objective function (sum of the travel distance and overtime costs) value related to this solution is equal to 27126.129 .

[Please insert Table 1 about here]

[Please insert Figure 1 about here]

[Please insert Figure 2 about here] 
In this article, the assumption of the understudied problem are as follows:

- Nurses start their routes from different health centers (initial depots) but finally return to a hospital (final depot). In fact, each nurse is assigned to one health center and starts his/her trip from it with essential equipment and medicines.

- Nurses visit several patients and finally deliver patients medical report, infectious needle and blood samples to a hospital.

- Each vehicle belongs to one transportation mode (private or public).

- Each nurse is assigned to exactly one vehicle and one mode of transportation.

- Every patient is assigned to a specific nurse based on the required service.

- Service times are considered for patients in different time intervals.

- The patients receive services within a hard time window.

- Traffic and unexpected events are not considered in the presented model.

\section{Mathematical model}

In this section, a MILP model is presented for the HHCRSP. The related sets, indices, parameters and variables are listed below. Then the mathematical formulation is presented.

\section{Indices}

$k \quad$ Index for vehicles

$n \quad$ Index for nurses

$i, j \quad$ Index for all the nodes

$f \quad$ Index for final depot (hospital)

\section{Sets}

$N \quad$ Set of nurses; $N=\left\{N_{1}, N_{2}, \ldots, N_{n_{\max }}\right\}$

$K \quad$ Set of vehicles; $K=\left\{K_{1}, K_{2}, \ldots, K_{k_{\max }}\right\}$

$U \quad$ Set of transportation mode; $U=\left\{u_{1}, u_{2}, \ldots, u_{k_{\max }}\right\}$

$V \quad$ Set of vertices which is included $\left\{V_{p} \cup V_{s} \cup V_{e}\right\}$

$V_{p} \quad$ Set of patients' vertices; $V_{p}=\left\{V_{p 1}, V_{p 2}, \ldots, V_{p_{n p_{\max }}}\right\}$

$V_{s} \quad$ Set of initial depots; $V_{s}=\left\{V_{S 1}, V_{S 2}, \ldots, V_{S_{n s_{\max }}}\right\}$

$V_{e} \quad$ Final depot set (set with one element)

$Q \quad$ Set of arcs 


\section{Parameters}

$s t_{i} \quad$ Service time for patient $i$

$n_{\max } \quad$ Number of nurses

$k_{\max } \quad$ Number of vehicles

$n s_{\max } \quad$ Number of initial depots (health centers)

$n p_{\max } \quad$ Number of patients

$\left[e_{i}, l_{i}\right] \quad$ Lower and upper bound of patient $i$ time window

$\left[a_{n}, b_{n}\right] \quad$ Lower and upper bound of nurse $n$ time window

$M \quad$ Big number

$c_{i, j, u_{(k)}} \quad$ Travel cost between nodes $i$ and $j$ by vehicle $k$ under mode $u_{(k)}$

$t_{i, j, u_{(k)}} \quad$ Travel time between nodes $i$ and $j$ by vehicle $k$ under mode $u_{(k)}$

$r_{n} \quad$ Maximum regular working time duration for each nurse $n$

$m_{n} \quad$ Maximum allowed daily working time duration for each nurse $n\left(r_{n} \leq m_{n}\right)$

$d_{n} \quad$ A cost of $d_{n}$ is incurred when working time is exceeded $r_{n}$

\section{Decision Variables:}

$Y_{i, j, n} \quad$ Binary variable taking value 1 if nurse $n$ travels from node $i$ to $j ; 0$, otherwise.

$X_{i, j, n, k} \quad$ Binary variable taking value 1 if the vehicle $k$ transfers nurse $n$ from node $i$ to $j$; , otherwise.

$W_{i} \quad$ Binary variable taking value 1 if waiting until beginning of time window is necessary; 0 , otherwise.

$Z_{n, k} \quad$ Binary variable taking value 1 if vehicle $k$ is assigned to nurse $n ; 0$, otherwise.

$T_{i} \quad$ Time at which nurse arrives at node $i\left(i \in V_{p}\right)$

$T_{i, n} \quad$ Time at which nurse $n$ leaves from initial depot $i\left(i \in V_{s}\right)$

$T_{f, n} \quad$ Time at which nurse $n$ arrives at final depot

$O_{n} \quad$ Amount of overtime done by nurse $n$

Using this information, the mathematical model for HHCRSP is as follows.

\section{Objective function:}

$\operatorname{Min} \sum_{(i, j, n) \in Q} \sum_{k \in K} c_{i, j, u} X_{i, j, n, k}+\sum_{n} d_{n} O_{n}$ 


\section{Constraints:}

$\sum_{i, n \mid(i, j, n) \in Q} Y_{i, j, n}=1$

$\forall j \in V_{p}$

$\sum_{i \in V_{S}} \sum_{j \mid(i, j, n) \in Q} Y_{i, j, n}=1$

$\forall n \in N$

$\sum_{i \mid(i, f, n) \in Q} Y_{i, f, n}=1$

$\forall n \in N$

$\sum_{j \mid(i, j, n) \in Q} Y_{i, j, n}=\sum_{j \mid(i, j, n) \in Q} Y_{j, i, n}$

$\forall i \in V_{p} ; n \in N$

$Y_{i, j, n}=\sum_{k \in K} X_{i, j, n, k}$

$\forall(i, j, n) \in Q$

$\sum_{n \in N} Z_{n, k} \leq 1$

$\forall k \in K$

$\sum_{k \in K} Z_{n, k} \leq 1$

$\forall n \in N$

$X_{i, j, n, k} \leq Z_{n, k}$

$\forall(i, j, n) \in Q ; k \in K$

$T_{i}+s t_{i}+\sum_{n \mid(i, j, n) \in Q} t_{i, j, u_{(k)}} X_{i, j, n, k} \leq T_{j}+M\left(1-\sum_{n \mid(i, j, n) \in Q} X_{i, j, n, k}\right) \quad \forall i, j \in V_{p} ; k \in K$

$T_{i, n}+t_{i, j, u_{(k)}} \leq T_{j}+M\left(1-X_{i, j, n, k}\right)$

$\forall(i, j, n) \in Q ; i \in V_{s} ; j \neq f ; k \in K$

$T_{i}+s t_{i}+t_{i, f, u_{(k)}} \leq T_{f, n}+M\left(1-X_{i, f, n, k}\right)$

$\forall(i, f, n) \in Q ; i \notin V_{s} ; k \in K$

$e_{i} \leq T_{i} \leq l_{i}$

$\forall i \in V_{p}$

$T_{j} \leq T_{i, n}+t_{i, j, u_{(k)}}+M\left(1-X_{i, j, n, k}\right)$

$\forall(i, j, n) \in Q ; i \in V_{s} ; j \in V_{p} ; k \in K$

$T_{f, n} \leq T_{i}+s t_{i}+t_{i, f, u_{(k)}}+M\left(1-X_{i, f, n, k}\right)$

$\forall(i, f, n) \in Q ; i \in V_{p} ; k \in K$

$T_{j} \leq T_{i}+s t_{i}+\sum_{n} \sum_{k} t_{i, j, u_{(k)}} X_{i, j, n, k}+M\left(1-\sum_{n} \sum_{k} X_{i, j, n, k}+W_{j}\right) \quad \forall i, j \in V_{p} \quad(i \neq j)$

$T_{j} \leq e_{j}+M\left(1-W_{j}\right)$

$\forall j \in V_{p}$

$a_{n} \leq T_{f, n} \leq b_{n}$

$\forall n \in N$ 
$a_{n} \leq T_{i, n} \leq b_{n}$

$T_{f, n}-T_{i, n} \leq m_{n}$

$O_{n} \geq T_{f, n}-T_{i, n}-r_{n}$

$Y_{i, j, n}, X_{i, j, n, k}, Z_{n, k}, W_{i}$ (binary variables) and $T_{i}, T_{i, n}, T_{f, n}, O_{n}$ (continuoues variables) $\geq 0$

Objective function (1) minimizes the sum of the travel distance and overtime costs. Constraint (2) ensures that each patient should be visited only once by a qualified nurse. Constraints (3) and (4) ensure that each nurse journey starts at the initial depots and ends at final depot. Constraint (5) confirms that if nurse $n$ enters a patient node then the nurse should leave that node to another allowed node. Constraint (6) shows the relationship between the $Y_{i, j, n}$ and $X_{i, j, n, k}$ decision variables. This constraint indicates that each nurse $n$ should travel from node $i$ to node $j$ by exactly one vehicle. Binary variable $Y_{i, j, n}$ taking value 1 if nurse $n$ travels from node $i$ to $j$; Also, binary variable $X_{i, j, n, k}$ taking value 1 if the vehicle $k$ transfers nurse $n$ from node $i$ to $j$. We used this constraint to establish reasonable relationship between these variables and ensure transportation of each nurse by only one vehicle. Constraints (7) and (8) ensure that each nurse use only one vehicle and also each vehicle is assigned to only one nurse. Binary variable $Z_{n, k}$ taking value 1 if vehicle $k$ is assigned to nurse $n$, so Constraint (9) states that if nurse $n$ travels from node $i$ to node $j$ by vehicle $k$, then nurse $n$ is definitely assigned to that vehicle. Because if vehicle $k$ is not assigned to nurse $n\left(Z_{n, k}=0\right)$, then $X_{i, j, n, k}=0$ and nurse $n$ cannot transfer from node $i$ to node $j$ by vehicle $k$. Constraint (10) indicates that the arrival time to node $j\left(j \in V_{p}\right)$ is greater or equal than the arrival time to node $i\left(i \in V_{p}\right)$ in addition to the service delivery time of node $i$ as well as travel time between two nodes. According to constraint (11), no flow is established directly from origin nodes to destination. Constraint (12) shows the accuracy of arriving time to final depot. Constraint (13) guarantees that each patient service delivery is started within its time window. Constraint (14) states that if nurse $n$ is moved between initial depot $i\left(i \in V_{s}\right)$ and node $j\left(j \in V_{p}\right)$ by vehicle $k$ under mode $u_{(k)}$, maximum arriving time to node $j$ is equal to time of leaving initial depot in addition to travel time between two nodes. Constraint (15) illustrates that maximum arriving time to final depot is equal to arriving time to the last patient node in addition to the time of service delivery in that node as well as travel time between the last patient and final depot. Based on constraint (16), maximum arriving time to node $j$ is equal to arriving time to previous node in addition to the time of service delivery in previous node as well as travel time between two nodes. In this constraint, if nurse $n$ travels from node $i$ to node $j$ by vehicle $k$ then $\sum_{n} \sum_{k} X_{i, j, n, k}$ is equal to 1 . Existence of big number $M$ becomes dependent on the existence of $W_{j}$. So, if waiting time 
for node $j$ exists $\left(W_{j}=1\right)$, big number $M$ disables the given constraint. But if there is no waiting time, this constraint works. According to constraint (17), if patient time window is started, waiting time is not allowed and the service must be done for the patient by the assigned nurse without delay. In fact, arriving time of the nurse to node $j\left(T_{j}\right)$ is limited up to patient time window because delay is a waste of time for both nurse and patient. So, if waiting is occurred, the activity can not to start later than time window and delay is not allowed. In fact, waiting times within the time window are prohibited by constraints (14-17). Constraint (18) and (19) make sure that caregivers are only allowed to work within a given time window. Constraint (20) ensures that maximum working time is not exceeded and constraint (21) shows the overtime calculation. Equations (22) represent the decision variables domain.

\section{Meta-heuristic approaches}

Due to computational complexity, solving medium and large-sized HHCRSP in reasonable time by exact methods is not possible. So, we have resorted to meta-heuristics to solve the problem. In the presented study, IWO [25], GOA [26] and SA [27] algorithms are presented for the optimization process. In the following, solution representation method, neighborhood structure operators, steps of utilized algorithms and parameter tuning of algorithms are described.

\subsection{Solution representation}

In this paper, the solutions are illustrated in a multiple string format. A separate string is provided for each nurse. Also, a vehicle string is considered wherein each nurse is assigned to one vehicle. In this new solution representation method, the initial depots of nurses, order of patients' visitation and the assigned vehicles are determined. To describe solution representation method, the feasible solution related to the defined problem of section 2 (as shown in Table 1) is considered. The representation of this solution is shown in Figure 3. This representation is included three strings for determining nurses' routs (nurses' strings) and one for assigning vehicle (Vehicle string). Two first cells of the nurses' strings show the two initial depots and the larger one in terms of value is selected as initial center of nurse. The third and fourth elements are corresponded to the patients who are assigned to the nurses. In decoding scheme, these two elements are arranged in ascending order and patients' visitation is done by the nurse according to it. Based on the first string of solution representation, nurse $N_{1}$ begins the route from initial depot $V_{s 1}$, visits $V_{p 1}$ and $V_{p 3}$ respectively and finally goes to the final depot $\left(V_{e}\right)$.

Similarly, the path of other nurses is presented as separated strings in Figure 3. Furthermore, vehicle allocation to nurse should be specified. In Figure 3, the fourth string of solution representation shows the allocation of vehicles to the nurses based on vehicle number. Nurses' routes related to above example is illustrated in Figure 4. In the presented example, number of patients who are assigned to all nurses is equal. But sometimes different numbers of patients are 
assigned to nurses. As a result, it is not possible to consider solution representation in a matrix form. For example, the representation of one feasible solution from a problem with the unbalanced allocation of nurses to the patients is shown in Figure 5. In this figure the length of strings is not the same. Nurses' routes related to example of Figure 5 is illustrated in Figure 6.

[Please insert Figure 3 about here]

[Please insert Figure 4 about here]

[Please insert Figure 5 about here]

[Please insert Figure 6 about here]

\subsection{Neighborhood structure}

To improve the quality of solutions, the neighborhood structure operator is used for generating neighboring solution from current solution. In this paper, four neighborhood structures, SwapNurse string operator, Regenerating-Nurse string operator, Swap-Vehicle string operator and Reordering-Vehicle string operator are presented for creating neighboring solutions. We profit from the operators' ideas presented by Nasir and Kuo [28] in developing the proposed neighborhood structures. To implement the neighborhood structures related to nurse string, one string among nurses' strings of the candidate solution is first chosen randomly. In Swap-Nurse string operator, two cells are selected from the nurse string at random and values of their locations are substituted. In Regenerating-Nurse string operator, one point of the nurse string is chosen randomly, then values from that point to the end of the string are regenerated. This operator may change the left or right hand side of the selected point randomly. Therefore, Initial depot of the nurse and the order of patients' visitation can be changed by these two operators. In Swap-Vehicle string operator, two cells are selected from the vehicle string at random and values of their locations are replaced. Furthermore, Reordering-Vehicle string operator is implemented for vehicle string which in one point of the string is chosen randomly, then values from that point to the end of the string are reordered. This operator may reorder the left or right hand side of the selected point at random. The procedure of these four neighborhood structures are shown in Figures 7, 8, 9 and 10. Notes that based on the solution representation method, processes of neighborhood structures and investigation of presented algorithms' results, it is turned out that the presented neighborhood structure operators does not produce infeasible neighboring solutions in the search procedure and the infeasibility test is not needed.

[Please insert Figure 7 about here]

[Please insert Figure 8 about here]

[Please insert Figure 9 about here]

[Please insert Figure 10 about here] 


\subsection{GOA algorithm}

Grasshoppers are herbivorous insects and pests of agricultural crops especially when they move as swarm and destroy entire crop fields. GOA method is a population-based meta-heuristic algorithm and inspired from the swarming behavior of grasshoppers in the nature. Nature-inspired meta-heuristic algorithms logically divide the search process into two main categories; exploration and exploitation. These two functions are implemented in GOA [26]. This algorithm has been used in many articles such as Rajkumar et al. [29] and Renukadevi and Karunakaran [30], but it has not been utilized in HHCRSP domain as far as we know. In GOA, the position of grasshopper $i$ in each iteration $\left(X_{i}\right)$ is determined by three factors. Social interaction $\left(S_{i}\right)$, gravity force $\left(G_{i}\right)$ and wind advection $\left(A_{i}\right)$. The position of $i$-th grasshopper during iterations is obtained by equation (23):

$$
X_{i}=S_{i}+G_{i}+A_{i}
$$

Wind advection $\left(A_{i}\right)$, the gravity force $\left(G_{i}\right)$ and the social interaction $\left(S_{i}\right)$ are calculated by the equations (24), (25) and (26):

$$
\begin{aligned}
A_{i} & =u \hat{e}_{w} \\
G_{i} & =-g \hat{e}_{g} \\
S_{i} & =\sum_{\substack{j=1 \\
j \neq i}}^{N} s\left(d_{i j}\right) \hat{d}_{i j}
\end{aligned}
$$

Where $\mathrm{u}$ is a constant drift, $\hat{e}_{w}$ is a unity vector in the direction of wind, $\mathrm{g}$ is the gravitational constant and $\hat{e}_{g}$ is a unity vector towards the center of earth. $d_{i j}$ is the distance between the grasshopper $i$ and $j$, s is a function to define the strength of social forces and $\hat{d}_{i j}$ is a unit vector from the $i$-th grasshopper to the $j$-th grasshopper. Social forces (s), distance between grasshoppers $\left(d_{i j}\right)$ and the unit vector $\left(\hat{d}_{i j}\right)$ are computed by the equations (27), (28) and (29):

$$
\begin{aligned}
& s(r)=f e^{\frac{-r}{l}}-e^{-r} \\
& d_{i j}=\left|x_{j}-x_{i}\right| \\
& \hat{d}_{i j}=\frac{x_{j}-x_{i}}{d_{i j}}
\end{aligned}
$$

Substituting $S, G$, and $A$ in equation (23), this equation can be rewritten as equation (30): 


$$
X_{i}=\sum_{\substack{j=1 \\ j \neq i}}^{N} s\left(\left|x_{j}-x_{i}\right|\right) \frac{x_{j}-x_{i}}{d_{i j}}-g \hat{e}_{g}+u \hat{e}_{w}
$$

Where $N$ is the number of grasshoppers. An improved version of this equation is shown as equation (31):

$$
X_{i}^{d}=c\left(\sum_{\substack{j=1 \\ j \neq i}}^{N} c \frac{u b_{d}-l b_{d}}{2} s\left(\left|x_{j}-x_{i}\right|\right) \frac{x_{j}-x_{i}}{d_{i j}}\right)+\hat{T}_{D}
$$

That $\hat{T}_{D}$ is the best solution found up to now, $u b_{d}$ and $l b_{d}$ are the upper bound and lower bound, and finally $\mathrm{c}$ is a decreasing coefficient [26]. Also, neighborhood structures presented in the section 4.2 are used to main diversity of solutions and change the position of the grasshoppers. For each candidate grasshopper, a uniform random number (i.e., $R \sim U(0,1)$ ) is generated. If $R<0.5$, the new neighboring solution is generated from the neighborhood of the current solution related to the candidate grasshopper. Otherwise, the current solution is unchanged. The pseudo code of the presented GOA is shown in Figure 11. The process of this algorithm will be continued until the stopping condition is met. GOA can be one of the suitable meta-heuristic algorithms to solve the HHCRSP. So, it is employed in this paper to solve the problem.

\section{[Please insert Figure 11 about here]}

\subsection{IWO Algorithm}

One of the meta-heuristics that can be used to solve combinatorial optimization problem such as HHCRSP is IWO algorithm that is introduced by Mehrabian and Lucas [25]. This method is a numerical stochastic optimization algorithm inspired by the fact of colonization of invasive weeds in nature based on weed biology and ecology [31]. IWO is an efficient and effective optimization tool by gradual evolution and inspired by the unique natural characteristics of weeds in seed production and cultivation. In this algorithm, firstly, main parameters of the algorithm are determined. Subsequently a certain number of seeds (initial solutions) are randomly distributed in the solution space with random positions (Initialization of a population), then each seed grows to a flowering plant and reaches the stage of seed producing (reproduction). The number of seeds that each plant is allowed to produce depends on its fitness, as well as maximum and minimum fitness of invasive weeds colony. The seed production procedure in the colony of weeds is shown in Figure 12.

\section{[Please insert Figure 12 about here]}

The produced seeds disperse normally over the search space near to the parent plant (spatial dispersal). Search diversification and randomness of the algorithm is presented in this part. The 
seeds are normally distributed with mean equal to zero. The standard deviation $(\delta)$ of the normal distribution function decreases at each step from the initially defined value $\left(\delta_{i}\right)$ to the final value $\left(\delta_{f}\right)$. The relationship between the above parameters and the standard deviation of each iteration can be expressed as equation (32):

$$
\delta_{\text {iter }}=\frac{\left(\text { iter }_{\max }-\text { iter }\right)^{n}}{\left(\text { iter }_{\max }\right)^{n}}\left(\delta_{f}-\delta_{i}\right)+\delta_{f}
$$

Where $\delta_{i t e r}$ is the standard deviation at the current step, iter ${ }_{\max }$ is the maximum number of iterations and $n$ is the nonlinear modulation index. In this paper, to restrict premature convergence or stick in local optima, neighborhood structure operators are applied on the produced seeds from each weed. For each candidate seed, a uniform random number (i.e., $R \sim U(0,1)$ ) is generated. If $R<0.5$, the new neighboring seed (neighboring solution) is generated from the neighborhood of the candidate seed by one of the presented neighborhood structure operators. Otherwise, the candidate seed is preferred. Then, new generated seeds are added to the colony. After passing some iterations, the number of plants in a colony reaches the maximum allowable population, $p_{\max }$, so only plants with better fitness can survive and produce seeds, others are being eliminated (competitive exclusion). In this step, the new generated seeds and their parent weeds are ranked and sorted together based on their fitness. From the existence plants, the maximum number of population are selected based on better fitness value and the rest of them will be removed (elimination mechanism). Survived plants are regenerated and repeat the above steps. The pseudo code of presented IWO is shown in Figure 13. The process of this algorithm will be continued until the stopping condition is met. IWO is one of the utilized meta-heuristic algorithms for solving the understudied problem in this article.

\section{[Please insert Figure 13 about here]}

\subsection{SA Algorithm}

SA algorithm is a powerful and widespread meta-heuristic algorithm for solving combinatorial optimization problems. In this method, optimization begins with a random solution $(S)$ and an initial temperature $\left(T_{0}\right)$ [27]. There is enough computational effort at each temperature with a search procedure. In each iteration of the search procedure, if the new generated neighboring solution is better than current solution, we will definitely accept the new solution according to Hill Climbing method. Otherwise, based on the Boltzmann function, we may accept the inferior neighboring solution. Neighborhood structure operators are used to generate neighboring solution from the current solution in the search procedure. After completion of the search procedure iterations, the temperature decreases based on a specific pattern. In this article, the following function is used to decrease the temperature:

$$
T_{i+1}=\alpha \times T_{i}
$$


Where $\alpha$ is a constant cooling rate. The search procedure will happen at each temperature again. This process is done until the stop criteria is met. The pseudo code of presented SA is shown in Figure 14. SA algorithm is also used in this article besides GOA and IWO to solve the HHCRSP problem.

\section{[Please insert Figure 14 about here]}

\subsection{Parameter tuning}

In this section Taguchi method [32] is used to statistically design the experiments as one of the best ways of the parameter setting. In this method, there is no need to investigate all factorials and only studying a part of experiments can lead the best level of each factor. The Taguchi method divides the factors into two main controllable and noise factors. This method seeks to minimize the effect of noise factors and to determine the optimal levels of controllable factors. The results of each experiment in the Taguchi method are converted to the "Signal-to-Noise $(S / N)$ " ratio. The purpose of these experiments is to find the levels that maximize the $S / N$ ratio. The $S / N$ ratio of the minimization objectives is computed by equation (34):

$$
S / N \text { ratio : }-10 \log _{10}\left(\frac{1}{n} \sum_{i=1}^{n} y_{i}^{2}\right) \quad[d b]
$$

where $y$ is the response variable and $n$ is the number of experiments. Also, the $S / N$ ratios are exposed in terms of the desi-bell $(d b)$ scale. In this paper, Taguchi method is used in parameter tuning to achieve better robustness of the three presented algorithms. For the GOA, IWO and SA algorithms three, five and two factors are considered, respectively. Table 2 shows the factors levels of GOA algorithm. Three controllable factors of GOA are: population size (NP), Maximum reduction of the search coverage $\left(c_{\max }\right)$ and minimum reduction of the search coverage $\left(c_{\min }\right)$. These factors are investigated on three levels. Five factor levels of IWO algorithm are illustrated in Table 3. Population size $(N P)$, minimum number of seeds $\left(S_{\min }\right)$, maximum number of seeds $\left(S_{\max }\right)$, initial value of the standard deviation $\left(\delta_{i}\right)$ and final value of the standard deviation $\left(\delta_{f}\right)$ are IWO algorithm factors which are measured in 3 levels. Table 4 shows the SA algorithm factors levels. Initial temperature $\left(T_{0}\right)$ and cooling rate $(\alpha)$ are two parameters of SA algorithm which are measured in three levels.

For parameter tuning, some test problems with different sizes and specifications are generated. Given the number and levels of each algorithm factors, the Minitab 16 software specifies the different combinations of factors levels in different trials. Tables 5, 6 and 7 shows the implemented different combinations of factors levels for parameter setting of three GOA, IWO, SA algorithms in $9\left(\mathrm{~L}_{9}\right), 27\left(\mathrm{~L}_{27}\right)$ and $9\left(\mathrm{~L}_{9}\right)$ runs, respectively. Then, Taguchi method is implemented and obtained results are presented. In this step, the stop conditions are the same for three algorithms and equal to allowed computational times. After obtaining the results of the test problems in different trials, the results of each trial are transformed into the $S / N$ ratio. The $S / N$ ratios of trials are averaged in each level, and its value is plotted against each control factor. The average $S / N$ ratio plot for GOA, IWO and SA algorithms are shown in Figures 15, 16 and 17, respectively. 
Since $S / N$ ratio with the higher value is better, so we selected the maximum point of each graph for related parameter to determine the optimal level. According to Figure 15 optimal values for $N P, c_{\max }$ and $c_{\min }$ are set in levels 3,2 and 3. Also based on Figure 16 optimal values for $N P$, $S_{\min }, S_{\max }, \delta_{i}$ and $\delta_{f}$ are set in levels 3,1, 3, 2 and 1. At last, according to Figure 17 optimal values for both $T_{0}$ and $\alpha$ are set in level 2. Tables 8, 9 and10 illustrate the optimal factors levels of GOA, IWO and SA algorithms, respectively.

[Please insert Table 2 about here]

[Please insert Table 3 about here]

[Please insert Table 4 about here]

[Please insert Table 5 about here]

[Please insert Table 6 about here]

[Please insert Table 7 about here]

[Please insert Figure 15 about here]

[Please insert Figure 16 about here]

[Please insert Figure 17 about here]

[Please insert Table 8 about here]

[Please insert Table 9 about here]

[Please insert Table 10 about here]

\section{Computational results}

This section describes the Numerical Experiments which is used to the validity of the proposed MILP model and compare the efficiencies of proposed algorithms. To conduct the experiments, we use a PC with $2.3 \mathrm{GHz}$ (Core I3) and $4 \mathrm{~GB}$ of RAM memory for performing computational study. The characteristics of 32 created random test problems are shown in Table 11. Problems $P_{1}$ to $\mathrm{P}_{16}$ are small-sized problems and $\mathrm{P}_{17}$ to $\mathrm{P}_{32}$ are medium and large-sized problems. In this Table symbol a/b/c/d shows the number of initial depots, number of nurses, number of private vehicles and the number of patients for each problem, respectively. The number of nurses is equal to the number of available vehicles. Therefore, the number of public vehicles is calculated by subtracting the number of private vehicles from the number of nurses. Moreover, interval values of parameters for generating random test problems are shown in Table 12. 
[Please insert Table 11 about here]

[Please insert Table 12 about here]

The computational results of solving 32 test problems are provided in Tables 13, 14 and 15. Table 13 shows the obtained results of exact method by GAMS software and three meta-heuristics for small sized-problems. It should be noted that 3600 seconds computational time limit as a stop criterion were considered to solve the small-sized problems by GAMS software. This stop condition is derived from the literature review of scheduling problem and determined based on a reasonable time to achieve an optimal solution by exact methods. Also, three presented metaheuristic algorithms were run for these problems within 400 seconds computational time limit as a stop criterion in MATLAB R2014a. In this table, the best MILP objective function value (the objective value of the best integer solution found by the GAMS within the given time limit) and CPU time of the software are reported. Also, each meta-heuristic was run five times for each problem in MATLAB and the best solution is illustrated in this Table. The results of solving sample problems $\mathrm{P}_{1}$ to $\mathrm{P}_{12}$ are the same in both exact and meta-heuristic methods and all of them have obtained optimal solutions. Problems $\mathrm{P}_{1}$ to $\mathrm{P}_{12}$ were solved within an acceptable computational time by GAMS sotware, but GAMS is not capable of solving problems with larger size. As can be seen in Table 13, obtaining optimal solution for $\mathrm{P}_{13}$ to $\mathrm{P}_{16}$ by GAMS software in 3600 seconds is not possible.

Also, Table 14 shows the results of proposed meta-heuristics for small sized problems in terms of $\overline{R P D}$ measure. Since five runs of algorithm are performed for each test problem, $R P D$ should be calculated for result of each run. Then mean $R P D$ results of runs $(\overline{R P D})$ is computed and used for making comparisons among algorithms. The RPD index [33] is calculated by the equation (35):

$R P D_{i j}=\frac{\text { Best }_{i j}-\min _{j} \text { Best }_{i j}}{\min _{j} \text { Best }_{i j}} \times 100$

Where, index $i$ shows the test problem number and index $j$ shows the used algorithms. Best $t_{i j}$ is the best solution value obtained from the algorithm $j$ for the problem $i$ and $\underset{j}{\min }$ Best $_{i j}$ is the best solution value obtained from the algorithms for the given problem. As indicated in Table 14, the best performance in small sizes is obtained by the IWO with the average $\overline{R P D}$ of $0.094 \%$.

Since HHCRSP belongs to NP-hard class, Problems $\mathrm{P}_{17}$ to $\mathrm{P}_{32}$ (medium and large-sized problems) were solved by meta-heuristics. GOA, IWO and SA algorithms were run within 1200 seconds computational time limit as a stop criterion in medium and large sizes. Stop condition of presented meta-heuristic algorithms are determined based on the computational study' results and convergence rate of the methods in finding better solution. Table 15 compare the performance of 
the presented algorithms in medium and large- sized problems in terms of $\overline{R P D}$ measure. According to Table 15, the best performance in medium and large-sized problems is obtained by the IWO with the average $\overline{R P D}$ of $1.446 \%$. Also, Figure 18 and 19 summarize the result of three presented algorithms based on the $\overline{R P D}$ criterion on small-sized problems and medium and largesized problems, respectively.

[Please insert Table 13 about here]

[Please insert Table 14 about here]

[Please insert Table 15 about here]

[Please insert Figure 18 about here]

[Please insert Figure 19 about here]

In order to statistical analysis of the proposed meta-heuristic algorithms, the Analysis of Variance (ANOVA) at 95\% confidence level is presented for variant size of problems separately. The purpose of ANOVA is to compare the means of several populations to determine if the population means are equal or there is a significant difference. Assumption $H_{0}$ states that the means' of three algorithms $\overline{R P D}$ are equal, and assumption $H_{1}$ expresses the mean inequality. It should be noted that the algorithm with better performance has lower $\overline{R P D}$ values. The obtained results by Minitab 16 are presented in Table 16 to Table 19. Table 16 and 17 presents ANOVA results for three meta-heuristic algorithms on small and medium-large sizes of problems. According to the obtained $p$-value (0.148) from Table 16, there is no significant difference among there presented algorithms for small-sized problems. But based on the calculated $p$-value (zero) from Table 17, the hypothesis $H_{0}$ is rejected and hypothesis $H_{1}$ is accepted. Therefore, there is significant difference among there presented algorithms for medium and large-sized problems.

Tukey test is a multiple comparison test and is utilized when more than two groups' means are being compared. This statistical test compares all possible pairs of means to determine which specific groups' means are different. Tables 18 and 19 show the results of Tukey test for small and medium-large sizes of problems, respectively. Also, Figures 20 and 21 show the output of Tukey's simultaneous 95\% confidence level. Result of Tukey test obtained from Table 18 and figure 20 confirms the result of Table 16. According to Table 18, all three algorithms are placed in one group (group A). Furthermore, according to Tukey pairwise comparisons plot for small-sized problems as shown in Figure 20, all three confidence intervals for the difference between the means of results' algorithms contain zero value. So, there are no significant differences between three algorithms for small-sized problems in terms of $\overline{R P D}$ measure. As shown in Table 19, IWO is placed in group $B$ and two other algorithms are placed in group $A$ for medium and large-sized 
problems. Also, based on the obtained results in Figure 21, confidence interval for the difference between SA and GOA contains zero value and the other difference confidence intervals doesn't contain zero value. Therefore, there is no significant difference between SA and GOA for medium and large-sized problems. The results indicate that IWO has better performance compared with two other algorithms based on Tukey test on medium and large-sized problems. At last, Figures 22 and 23 show the mean interval plot of algorithms on small and medium-large sizes of problems in terms of $\overline{R P D}$ measure. As depicted in Figure 22 and 23 mean results of IWO is at a lower level compared to SA and GOA means.

[Please insert Table 16 about here]

[Please insert Table 17 about here]

[Please insert Table 18 about here]

[Please insert Table 19 about here]

[Please insert Figure 20 about here]

[Please insert Figure 21 about here]

[Please insert Figure 22 about here]

[Please insert Figure 23 about here]

\section{Conclusions and future works}

In the presented study, a MILP model was proposed to determine both scheduling and routing of the nurses in a HHC problem. In this multi-depot HHCRSP with multimodal transportation, nurses start their routes from different health centers (initial depots) and return to a hospital (final depot), finally. Also, each nurse has a pre-selected set of patients who should only visit them. Each vehicle has belonged to one transportation mode (private or public) and each nurse has assigned to exactly one vehicle. The objective function of this problem was the sum of the travel distance and overtime costs minimization. The small-sized problems were solved by GAMS software. In addition, due to handle computational complexity, three meta-heuristics were proposed to solve the problem in medium and large-sized problems. In this paper, GOA, IWO and SA algorithms were utilized in the optimization process. A novel solution representation method for HHCRCP is presented in this study. In the solution representation method, the initial depots of nurses, order of patients' visitation and the assigned vehicles were determined. Also, the Taguchi method was used to statistically adjust the parameters and operators of the proposed algorithms. To verify the validity of the presented mathematical model and evaluate the presented algorithms, we have performed the computational study with 32 randomly generated test problems. According to the computational results, it can be concluded that the IWO algorithm had better performance in comparison to other algorithms. In the following, some suggestions are offered for future studies: 
- Studying a multi objective HHCRSP by concentrating on the related factors to sustainability.

- Considering uncertainty context to expansion of the problem model.

\section{References}

1. Decerle, J., Grunder, O., El Hassani, A.H. and Barakat, O. "A matheuristic for a multi-depot home health care problem", IFAC-PapersOnLine. 51(11), pp. 340-345 (2018).

2. Fathollahi-Fard, A.M., Govindan, K., Hajiaghaei-Keshteli, M. and Ahmadi, A. "A green home health care supply chain: New modified simulated annealing algorithms", Journal of Cleaner Production, 240, pp. 118200 (2019).

3. Rest, K.D., Trautsamwieser, A. and Hirsch, P. "Trends and risks in home health care". Journal of Humanitarian Logistics and Supply Chain Management, 2(1), pp. 34-53 (2019).

4. Fernandez, A., Gregory, G., Hindle, A. and Lee, A.C. "A model for community nursing in a rural county". Journal of the Operational Research Society, 25(2), pp. 231-239 (1974).

5. $\quad$ Begur, S.V., Miller, D.M. and Weaver, J.R. "An integrated spatial DSS for scheduling and routing home-health-care nurses". Interfaces, 27(4), pp. 35-48 (1997).

6. Braekers, K., Hartl, R.F., Parragh, S.N. and Tricoire, F. "A bi-objective home care scheduling problem: Analyzing the trade-off between costs and client inconvenience", European Journal of Operational Research, 248(2), pp. 428-443 (2016).

7. Fikar, C. and Hirsch, P. "Evaluation of trip and car sharing concepts for home health care services", Flexible Services and Manufacturing Journal, 30(1-2), pp. 78-97 (2018).

8. Nasir, J.A., and Dang, C. "Solving a more flexible home health care scheduling and routing problem with joint patient and nursing staff selection", Sustainability, 10(1), 148 (2018).

9. Fathollahi-Fard, A.M., Hajiaghaei-Keshteli, M. and Tavakkoli-Moghaddam, R. "A bi-objective green home health care routing problem”, Journal of Cleaner Production, 200, pp. $423-443$ (2018).

10. Tohidifard, M., Tavakkoli-Moghaddam, R., Navazi, F. and Partovi M. "A Multi-Depot Home Care Routing Problem with Time Windows and Fuzzy Demands Solving by Particle Swarm Optimization and Genetic Algorithm", IFAC-PapersOnLine, 51(11), pp. 358-363 (2018).

11. Lanzarone, E. and Matta, A. "Robust nurse-to-patient assignment in home care services to minimize overtimes under continuity of care", Operations Research for Health Care, 3(2), pp. 4858 (2014).

12. Hiermann, G., Prandtstetter, M., Rendl, A., Puchinger, J. and Raidl, G.R. "Metaheuristics for solving a multimodal home-healthcare scheduling problem", Central European Journal of Operations Research, 23(1), pp. 89-113 (2015).

13. Rest, K.D. and Hirsch, P. "Daily scheduling of home health care services using time-dependent public transport", Flexible Services and Manufacturing Journal. 28(3), pp. 495-525 (2016).

14. Xiao, Y. and Konak, A. "The heterogeneous green vehicle routing and scheduling problem with time-varying traffic congestion". Transportation Research Part E: Logistics and Transportation Review. 88: pp. 146-166 (2016).

15. Jakob, A., Craig, J.L. and Fisher, G. "Transport cost analysis: a case study of the total costs of private and public transport in Auckland". Environmental Science \& Policy. 9(1): pp. 55-66 (2006).

16. Masanobu, K.I.I. and Hanaoka, S. "Comparison of sustainability between private and public transport considering urban structure", IATSS Research, 27(2), pp. 6-15 (2003). 
17. Trautsamwieser, A., Gronalt, M. and Hirsch, P. "Securing home health care in times of natural disasters", OR spectrum, 33(3), pp. 787-813 (2011).

18. Liu, R., Yuan, B. and Jiang, Z. "Mathematical model and exact algorithm for the home care worker scheduling and routing problem with lunch break requirements", International Journal of Production Research, 55(2), pp. 558-575 (2017).

19. Riazi, S., Wigström, O., Bengtsson, K. and Lennartson, B. "A column generation-based gossip algorithm for home healthcare routing and scheduling problems", IEEE Transactions on Automation Science and Engineering, 16(1), pp.127-137 (2018).

20. Euchi, J. "Optimising the routing of home health caregivers: can a hybrid ant colony metaheuristic provide a solution?", British Journal of Healthcare Management, 26(7), pp. 192-196 (2020).

21. Shi, Y., Boudouh, T. and Grunder, O. "A hybrid genetic algorithm for a home health care routing problem with time window and fuzzy demand", Expert Systems with Applications, 72, pp. 160-176 (2017).

22. Decerle, J., Grunder, O., El Hassani, A.H. and Barakat, O. "Impact analysis of workload balancing on the home health care routing and scheduling problem", in 2017 4th International Conference on Control, Decision and Information Technologies (CoDIT). IEEE (2017).

23. Mankowska, D.S., Meisel, F. and Bierwirth, C. "The home health care routing and scheduling problem with interdependent services". Health care management science. 17(1), pp. 15-30 (2014).

24. Liu, R., Tao, Y. and Xie, X. "An adaptive large neighborhood search heuristic for the vehicle routing problem with time windows and synchronized visits", Computers \& Operations Research, 101, pp. 250-262 (2019).

25. Mehrabian, A.R. and Lucas, C. " $A$ novel numerical optimization algorithm inspired from weed colonization", Ecological informatics, 1(4), pp. 355-366 (2006).

26. Saremi, S., Mirjalili, S. and Lewis, A. "Grasshopper optimisation algorithm: theory and application", Advances in Engineering Software. 105: pp. 30-47 (2017).

27. Kirkpatrick, S., Gelatt, C.D. and Vecchi, M.P. "Optimization by simulated annealing” science. 220(4598), pp. 671-680 (1983).

28. Nasir, J.A., and Kuo, Y.H. "A decision support framework for home health care transportation with simultaneous multi-vehicle routing and staff scheduling synchronization", Decision Support Systems, 138, 113361 (2020).

29. Rajkumar, R., Anandakumar, K. and Bharathi, A. "Grasshopper Optimization Algorithm based Feature Selection with Twin Support Vector Machine Classifier for Coronary Artery Heart Disease Prediction", International Journal of Control and Automation, 12(6), pp. 256-267 (2019).

30. Renukadevi, T. and Karunakaran, S. "Optimizing deep belief network parameters using grasshopper algorithm for liver disease classification". International Journal of Imaging Systems and Technology, 30(1), pp. 168-184 (2020).

31. Esmaeili-Douki, A., Mahzouni-Sani, M., Nikhalat Jahromi, A., and Jolai, F. “A novel fuzzy biobjective vehicle routing and scheduling problem with time window constraint for a distribution system: A case study", Scientia Iranica, In press (2020).

32. Ross, P.J. Taguchi Techniques for Quality Engineering: Loss Function, Orthogonal Experiments, Parameter and Tolerance Design. McGraw-Hill, New York (1988).

33. Yazdani, M., Zandieh, M., Tavakkoli-Moghaddam, R. and Jolai, F. "Two meta-heuristic algorithms for the dual-resource constrained flexible job-shop scheduling problem”, Scientia Iranica, 22(3), pp. 1242- 1257 ( 2015). 


\section{Biographies}

Fahime Ghiasvnd Ghiasi accomplished his M.Sc. in Industrial Engineering at Urmia University of technology, Urmia, Iran. Currently, she is a Ph.D. candidate in Industrial Engineering at Islamic Azad University, Qazvin branch, Iran. Her research interests are vehicle routing and scheduling problems especially home health care routing and scheduling problem.

Mehdi Yazdani accomplished his B.Sc. in Industrial Engineering at Islamic Azad University, Qazvin Branch, Iran (2002-2006), and M.Sc. in Industrial Engineering at Islamic Azad University, Qazvin Branch, Iran (2006-2008). He obtained his Ph.D. degree in Industrial Engineering from Islamic Azad University, Tehran Science and Research Branch, Iran (2010-2014). He is currently an Assistant Professor at the Department of Industrial Engineering, Islamic Azad University, Qazvin Branch, Iran. His research interests are Production Planning and Scheduling, Project Management, Applied Operations Research and Artificial Intelligence techniques.

Behnam Vahdani is currently a Post-Doctoral Research Fellow at IMT Atlantique, Lab-STICC, UBL, Brest, France. He is also an Associate Professor at the Department of Industrial Engineering, Qazvin Branch, Islamic Azad University. He received a Ph.D. degree from the School of Industrial Engineering at University of Tehran, Iran, and is currently a member of Iran's National Elite Foundation. His main research interests include the application of operations research, uncertainty programming, and machine learning.

Abolfazl Kazemi is currently an Associate Professor in Department of Industrial Engineering at Qazvin Islamic Azad University (QIAU). He received his B.Sc. in Industrial Engineering from Qazvin Islamic Azad University, his Master's degree in Industrial Engineering from Tarbiat Modares University (TMU) and his Ph.D. degree in Industrial Engineering from Amirkabir University of Technology (AUT). His research interests are in the areas of Data-driven Optimization, Business intelligence, Data Mining, Process Mining, Machine Learning, Intelligent Supply Chain, and Fuzzy Set Theory.

\section{Table Captions:}

Table 1. Sample problem parameters

Table 2. Factors levels of Grasshopper Optimization algorithm

Table 3. Factors levels of Invasive Weed Optimization algorithm

Table 4. Factors levels of Simulated Annealing algorithm

Table 5. Different combinations of factor levels in parameter setting of Grasshopper Optimization algorithm (L9) 
Table 6. Different combinations of factor levels in parameter setting of Invasive Weed Optimization algorithm $\left(\mathrm{L}_{27}\right)$

Table 7. Different combinations of factor levels in parameter setting of Simulated Annealing algorithm ( 99$)$

Table 8. Optimal levels and values of Grasshopper Optimization algorithm factors

Table 9. Optimal levels and values of Invasive Weed Optimization algorithm factors

Table 10. Optimal levels and values of Simulated Annealing algorithm factors

Table 11. Characteristics of the test problems

Table 12. Values of parameters

Table 13. The results on small-sized problems

Table 14. Results of $\overline{R P D}$ for the presented meta-heuristic algorithms on small-sized problems

Table 15. Results of $\overline{R P D}$ for the presented meta-heuristic algorithms on medium and large-sized problems

Table 16. ANOVA test results for the presented meta-heuristic algorithms on small-sized problems

Table 17. ANOVA test results for the presented meta-heuristic algorithms on medium and largesized problems

Table 18. Tukey test for comparison of the presented meta-heuristic algorithms at $95 \%$ confidence (small sizes)

Table 19. Tukey test for comparison of the presented meta-heuristic algorithms at $95 \%$ confidence (medium and large sizes)

\section{Figure Captions:}

Figure 1. Directed graph of nurses' routes

Figure 2. Gantt chart for nurses and patients

Figure 3. Representation of a solution

Figure 4. Nurses' routes related to Figure 3

Figure 5. Solution representation with the different length of strings

Figure 6. Nurses' routes related to Figure 5

Figure 7. The procedure of Swap - Nurse string operator 
Figure 8. The procedure of Regenerating - Nurse string operator

Figure 9. The procedure of Swap - Vehicle string operator

Figure 10. The procedure of Reordering - Vehicle string operator

Figure 11. Steps of the presented Grasshopper Optimization algorithm for the HHCRSP

Figure 12. Procedure of producing seed [25]

Figure 13. Steps of the presented Invasive Weed Optimization algorithm for the HHCRSP

Figure 14. Steps of the presented Simulated Annealing algorithm for the HHCRSP

Figure 15. The average $\mathrm{S} / \mathrm{N}$ ratio plot at each level of the factors for objective function values in Grasshopper Optimization algorithm

Figure 16. The average $\mathrm{S} / \mathrm{N}$ ratio plot at each level of the factors for objective function values in Invasive Weed Optimization algorithm

Figure 17. The average $\mathrm{S} / \mathrm{N}$ ratio plot at each level of the factors for objective function values in Simulated Annealing algorithm

Figure 18. Results of the presented meta-heuristic algorithms on small-sized problems

Figure 19. Results of the presented meta-heuristic algorithms on medium and large-sized problems Figure 20. Tukey pairwise comparisons plot of the presented meta-heuristic algorithms (small sizes)

Figure 21. Tukey pairwise comparisons plot of the presented meta-heuristic algorithms (medium and large sizes)

Figure 22. Mean interval plot of the presented meta-heuristic algorithms in terms of $\overline{R P D}$ measure (small sizes)

Figure 23. Mean interval plot of the presented meta-heuristic algorithms in terms of $\overline{R P D}$ measure (medium and large sizes)

\section{Tables:}

Table 1. Sample problem parameters

\begin{tabular}{ccccccc}
\hline Parameters & {$\left[\boldsymbol{a}_{\boldsymbol{n}}, \boldsymbol{b}_{\boldsymbol{n}}\right]$} & $\boldsymbol{e}_{\boldsymbol{i}}$ & $\boldsymbol{l}_{\boldsymbol{i}}$ & $\boldsymbol{r}_{\boldsymbol{n}}$ & $\boldsymbol{m}_{\boldsymbol{n}}$ & $\boldsymbol{s t}_{\boldsymbol{i}}$ \\
\hline Values & {$[0,480]$} & $\sim U[0,60]$ & $\sim U[420,480]$ & 360 & 480 & $\sim U[30,100]$ \\
\hline
\end{tabular}


Table 2. Factors levels of Grasshopper Optimization algorithm

\begin{tabular}{ccccc}
\hline Factors & Description & Level 1 & Level 2 & Level 3 \\
\hline $\boldsymbol{N P}$ & Population size & 100 & 150 & 200 \\
$\boldsymbol{c}_{\boldsymbol{m a x}}$ & Maximum reduction of the search coverage & 1 & 1.25 & 1.5 \\
$\boldsymbol{c}_{\boldsymbol{m i n}}$ & Minimum reduction of the search coverage & 0.0001 & 0.0002 & 0.0003 \\
\hline
\end{tabular}

Table 3. Factors levels of Invasive Weed Optimization algorithm

\begin{tabular}{ccccc}
\hline Factors & Description & Level 3 & Level 2 & Level 3 \\
\hline $\boldsymbol{N P}$ & Population size & 100 & 150 & 200 \\
$\boldsymbol{S}_{\boldsymbol{m i n}}$ & Minimum number of seeds & 1 & 2 & 3 \\
$\boldsymbol{S}_{\boldsymbol{m a x}}$ & Maximum number of seeds & 3 & 5 & 7 \\
$\boldsymbol{\delta}_{\boldsymbol{i}}$ & Initial value of the standard deviation & 0.03 & 0.05 & 0.07 \\
$\boldsymbol{\delta}_{\boldsymbol{f}}$ & Final value of the standard deviation & 0.0010 & 0.0015 & 0.0020 \\
\hline
\end{tabular}

Table 4. Factors levels of Simulated Annealing algorithm

\begin{tabular}{llccc}
\hline Factors & Description & Level 1 & Level 2 & Level 3 \\
\hline $\boldsymbol{T}_{\boldsymbol{0}}$ & Initial temperature & 20 & 25 & 30 \\
$\boldsymbol{\alpha}$ & Cooling rate & 0.85 & 0.90 & 0.95 \\
\hline
\end{tabular}

Table 5. Different combinations of factor levels in parameter setting of Grasshopper Optimization Algorithm $\left(\mathrm{L}_{9}\right)$

\begin{tabular}{cccc}
\hline \multirow{2}{*}{ Trial } & \multicolumn{3}{c}{ Level of control factors } \\
\cline { 2 - 4 } & $\boldsymbol{N P}$ & $\boldsymbol{c}_{\boldsymbol{m a x}}$ & $\boldsymbol{c}_{\boldsymbol{m i n}}$ \\
\hline 1 & 1 & 1 & 1 \\
2 & 1 & 2 & 2 \\
3 & 1 & 3 & 3 \\
4 & 2 & 1 & 2 \\
5 & 2 & 2 & 3 \\
6 & 2 & 3 & 1 \\
7 & 3 & 1 & 3 \\
8 & 3 & 2 & 1 \\
9 & 3 & 3 & 2 \\
\hline
\end{tabular}


Table 6. Different combinations of factor levels in parameter setting of Invasive Weed Optimization algorithm ( $\left.\mathrm{L}_{27}\right)$

\begin{tabular}{|c|c|c|c|c|c|}
\hline \multirow[b]{2}{*}{ Trial } & \multicolumn{5}{|c|}{ Level of control factors } \\
\hline & $N P$ & $S_{\min }$ & $S_{\max }$ & $\delta_{i}$ & $\delta_{f}$ \\
\hline 1 & 1 & 1 & 1 & 1 & 1 \\
\hline 2 & 1 & 1 & 1 & 1 & 2 \\
\hline 3 & 1 & 1 & 1 & 1 & 3 \\
\hline 4 & 1 & 2 & 2 & 2 & 1 \\
\hline 5 & 1 & 2 & 2 & 2 & 2 \\
\hline 6 & 1 & 2 & 2 & 2 & 3 \\
\hline 7 & 1 & 3 & 3 & 3 & 1 \\
\hline 8 & 1 & 3 & 3 & 3 & 2 \\
\hline 9 & 1 & 3 & 3 & 3 & 3 \\
\hline 10 & 2 & 1 & 2 & 3 & 1 \\
\hline 11 & 2 & 1 & 2 & 3 & 2 \\
\hline 12 & 2 & 1 & 2 & 3 & 3 \\
\hline 13 & 2 & 2 & 3 & 1 & 1 \\
\hline 14 & 2 & 2 & 3 & 1 & 2 \\
\hline 15 & 2 & 2 & 3 & 1 & 3 \\
\hline 16 & 2 & 3 & 1 & 2 & 1 \\
\hline 17 & 2 & 3 & 1 & 2 & 2 \\
\hline 18 & 2 & 3 & 1 & 2 & 3 \\
\hline 19 & 3 & 1 & 3 & 2 & 1 \\
\hline 20 & 3 & 1 & 3 & 2 & 2 \\
\hline 21 & 3 & 1 & 3 & 2 & 3 \\
\hline 22 & 3 & 2 & 1 & 3 & 1 \\
\hline 23 & 3 & 2 & 1 & 3 & 2 \\
\hline 24 & 3 & 2 & 1 & 3 & 3 \\
\hline 25 & 3 & 3 & 2 & 1 & 1 \\
\hline 26 & 3 & 3 & 2 & 1 & 2 \\
\hline 27 & 3 & 3 & 2 & 1 & 3 \\
\hline
\end{tabular}

Table 7. Different combinations of factor levels in parameter setting of Simulated Annealing algorithm $\left(\mathrm{L}_{9}\right)$

\begin{tabular}{ccc}
\hline \multirow{2}{*}{ Trial } & \multicolumn{2}{c}{ Level of control factors } \\
\cline { 2 - 3 } & $\boldsymbol{T}_{\mathbf{0}}$ & $\boldsymbol{\alpha}$ \\
\hline 1 & 1 & 1 \\
2 & 1 & 2 \\
3 & 1 & 3 \\
4 & 2 & 1 \\
5 & 2 & 2 \\
6 & 2 & 3 \\
7 & 3 & 1 \\
8 & 3 & 2 \\
9 & 3 & 3 \\
\hline
\end{tabular}


Table 8. Optimal levels and values of Grasshopper Optimization Algorithm factors

\begin{tabular}{cccc}
\hline Factors & Description & Optimal value & Optimal level \\
\hline $\boldsymbol{N P}$ & Population size & 200 & Level 3 \\
$\boldsymbol{c}_{\boldsymbol{m a x}}$ & Maximum reduction of the search coverage & 1.25 & Level 2 \\
$\boldsymbol{c}_{\boldsymbol{m i n}}$ & Minimum reduction of the search coverage & 0.0003 & Level 3 \\
\hline
\end{tabular}

Table 9. Optimal levels and values of Invasive Weed Optimization algorithm factors

\begin{tabular}{cccc}
\hline Factors & Description & Optimal value & Optimal level \\
\hline $\boldsymbol{N P}$ & Population size & 200 & Level 3 \\
$\boldsymbol{S}_{\boldsymbol{m i n}}$ & Minimum number of seeds & 1 & Level 1 \\
$\boldsymbol{S}_{\boldsymbol{m a x}}$ & Maximum number of seeds & 7 & Level 3 \\
$\boldsymbol{\delta}_{\boldsymbol{i}}$ & Initial value of the standard deviation & 0.05 & Level 2 \\
$\boldsymbol{\delta}_{\boldsymbol{f}}$ & Final value of the standard deviation & 0.001 & Level 1
\end{tabular}

Table 10. Optimal levels and values of Simulated Annealing algorithm factors

\begin{tabular}{cccc}
\hline Factors & Description & Optimal value & Optimal level \\
\hline $\boldsymbol{T}_{\boldsymbol{0}}$ & Initial temperature & 25 & Level 2 \\
$\boldsymbol{\alpha}$ & Cooling rate & 0.9 & Level 2 \\
\hline
\end{tabular}


Table 11. Characteristics of the test problems

\begin{tabular}{|c|c|c|c|c|c|c|}
\hline \multirow{2}{*}{$\begin{array}{c}\text { Test } \\
\text { problem } \\
\text { number }\end{array}$} & \multirow{2}{*}{$\begin{array}{l}\text { Number of } \\
\text { initial depots }\end{array}$} & \multirow{2}{*}{$\begin{array}{l}\text { Number } \\
\text { of nurses }\end{array}$} & \multicolumn{2}{|c|}{$\begin{array}{c}\begin{array}{c}\text { Number of } \\
\text { vehicles }\end{array} \\
\end{array}$} & \multirow{2}{*}{$\begin{array}{c}\text { Number of } \\
\text { patients }\end{array}$} & \multirow{2}{*}{ Symbol } \\
\hline & & & Private & Public & & \\
\hline $\mathrm{P}_{1}$ & 1 & 2 & 1 & 1 & 4 & $1 / 2 / 1 / 4$ \\
\hline $\mathrm{P}_{2}$ & 2 & 2 & 1 & 1 & 5 & $2 / 2 / 1 / 5$ \\
\hline $\mathrm{P}_{3}$ & 2 & 2 & 1 & 1 & 6 & $2 / 2 / 1 / 6$ \\
\hline $\mathrm{P}_{4}$ & 2 & 2 & 1 & 1 & 7 & $2 / 2 / 1 / 7$ \\
\hline $\mathrm{P}_{5}$ & 2 & 2 & 1 & 1 & 8 & $2 / 2 / 1 / 8$ \\
\hline $\mathrm{P}_{6}$ & 2 & 2 & 1 & 1 & 9 & $2 / 2 / 1 / 9$ \\
\hline $\mathrm{P}_{7}$ & 2 & 3 & 1 & 2 & 10 & $2 / 3 / 1 / 10$ \\
\hline $\mathrm{P}_{8}$ & 2 & 3 & 2 & 1 & 11 & $2 / 3 / 2 / 11$ \\
\hline $\mathrm{P}_{9}$ & 2 & 3 & 2 & 1 & 12 & $2 / 3 / 2 / 12$ \\
\hline $\mathrm{P}_{10}$ & 2 & 4 & 2 & 2 & 13 & $2 / 4 / 2 / 13$ \\
\hline $\mathrm{P}_{11}$ & 3 & 4 & 2 & 2 & 14 & $3 / 4 / 2 / 14$ \\
\hline $\mathrm{P}_{12}$ & 3 & 4 & 2 & 2 & 15 & $3 / 4 / 2 / 15$ \\
\hline $\mathrm{P}_{13}$ & 3 & 6 & 3 & 3 & 16 & $3 / 6 / 3 / 16$ \\
\hline $\mathrm{P}_{14}$ & 3 & 7 & 3 & 4 & 18 & $3 / 7 / 3 / 18$ \\
\hline $\mathrm{P}_{15}$ & 4 & 8 & 3 & 5 & 20 & $4 / 8 / 3 / 20$ \\
\hline $\mathrm{P}_{16}$ & 4 & 8 & 3 & 5 & 22 & $4 / 8 / 3 / 22$ \\
\hline $\mathrm{P}_{17}$ & 5 & 10 & 4 & 6 & 24 & $5 / 10 / 4 / 24$ \\
\hline $\mathrm{P}_{18}$ & 5 & 11 & 4 & 7 & 26 & $5 / 11 / 4 / 26$ \\
\hline $\mathrm{P}_{19}$ & 5 & 12 & 5 & 7 & 28 & $5 / 12 / 5 / 28$ \\
\hline $\mathrm{P}_{20}$ & 6 & 13 & 5 & 8 & 30 & $6 / 13 / 5 / 30$ \\
\hline $\mathrm{P}_{21}$ & 6 & 13 & 5 & 8 & 43 & $6 / 13 / 5 / 43$ \\
\hline $\mathrm{P}_{22}$ & 6 & 13 & 6 & 7 & 46 & $6 / 13 / 6 / 46$ \\
\hline $\mathrm{P}_{23}$ & 7 & 15 & 6 & 9 & 51 & $7 / 15 / 6 / 51$ \\
\hline $\mathrm{P}_{24}$ & 7 & 16 & 6 & 10 & 52 & $7 / 16 / 6 / 52$ \\
\hline $\mathrm{P}_{25}$ & 7 & 17 & 7 & 10 & 53 & $7 / 17 / 7 / 53$ \\
\hline $\mathrm{P}_{26}$ & 8 & 19 & 7 & 12 & 55 & $8 / 19 / 7 / 55$ \\
\hline $\mathrm{P}_{27}$ & 8 & 21 & 7 & 14 & 64 & $8 / 21 / 7 / 64$ \\
\hline $\mathrm{P}_{28}$ & 8 & 21 & 8 & 13 & 66 & $8 / 21 / 8 / 66$ \\
\hline $\mathrm{P}_{29}$ & 9 & 21 & 8 & 13 & 68 & $9 / 21 / 8 / 68$ \\
\hline $\mathrm{P}_{30}$ & 9 & 22 & 8 & 14 & 69 & $9 / 22 / 8 / 69$ \\
\hline $\mathrm{P}_{31}$ & 9 & 23 & 9 & 14 & 74 & $9 / 23 / 9 / 74$ \\
\hline $\mathrm{P}_{32}$ & 9 & 24 & 9 & 15 & 74 & $9 / 24 / 9 / 74$ \\
\hline
\end{tabular}


Table 12. Values of parameters

\begin{tabular}{lcc}
\hline \multicolumn{1}{c}{ Parameters } & Symbol & Random interval \\
\hline Coordinates of points & $\left(X C_{i}, Y C_{i}\right)$ & $\sim U[0,100]$ \\
Distance between each two nodes & $d_{i j}$ & $\sqrt{\left(X C_{i}-X C_{j}\right)^{2}+\left(Y C_{i}-Y C_{j}\right)^{2}}$ \\
Travel cost & $c_{i, j, u_{(k)}}$ & $\sim U[2,4]^{*} d_{i j}$ \\
Travel time & $t_{i, j, u_{(k)}}$ & $\sim U[1,1.5]^{*} d_{i j}$ \\
Service time & $s t_{i}$ & $\sim U[5,12]$ \\
Start of patient time window & $e_{i}$ & $\sim U[0,150]$ \\
End of patient time window & $l_{i}$ & $\sim U[400,550]$ \\
Regular working time & $r_{n}$ & 540 \\
Maximum working time & $m_{n}$ & 600 \\
Overtime cost & $d_{n}$ & $\sim U[10,30]$ \\
Start of nurse time window & $a_{n}$ & $\sim U[0,120]$ \\
End of nurse time window & $b_{n}$ & $\sim U[300,660]$ \\
\hline
\end{tabular}

Table 13. The results on small-sized problems

\begin{tabular}{ccccccc}
\hline \multicolumn{2}{c}{ Problem } & \multicolumn{2}{c}{ Results of GAMS } & \multicolumn{3}{c}{ Results of meta-heuristics } \\
\hline Problem No. & Symbol & OFV & CPU time & Best GOA & Best IWO & Best SA \\
\hline $\mathrm{P}_{1}$ & $1 / 2 / 1 / 4$ & 965.20 & 0.13 & 965.20 & 965.20 & 965.20 \\
$\mathrm{P}_{2}$ & $2 / 2 / 1 / 5$ & 781.17 & 0.28 & 781.17 & 781.17 & 781.17 \\
$\mathrm{P}_{3}$ & $2 / 2 / 1 / 6$ & 850.74 & 0.14 & 850.74 & 850.74 & 850.74 \\
$\mathrm{P}_{4}$ & $2 / 2 / 1 / 7$ & 749.16 & 0.16 & 749.16 & 749.16 & 749.16 \\
$\mathrm{P}_{5}$ & $2 / 2 / 1 / 8$ & 931.72 & 0.30 & 931.72 & 931.72 & 931.72 \\
$\mathrm{P}_{6}$ & $2 / 2 / 1 / 9$ & 869.28 & 0.34 & 869.28 & 869.28 & 869.28 \\
$\mathrm{P}_{7}$ & $2 / 3 / 1 / 10$ & 1414.39 & 31.07 & 1414.39 & 1414.39 & 1414.39 \\
$\mathrm{P}_{8}$ & $2 / 3 / 2 / 11$ & 1038.15 & 5.36 & 1038.15 & 1038.15 & 1038.15 \\
$\mathrm{P}_{9}$ & $2 / 3 / 2 / 12$ & 1439.92 & 12.56 & 1439.92 & 1439.92 & 1439.92 \\
$\mathrm{P}_{10}$ & $2 / 4 / 2 / 13$ & 1730.97 & 142.80 & 1730.97 & 1730.97 & 1730.97 \\
$\mathrm{P}_{11}$ & $3 / 4 / 2 / 14$ & 1931.06 & 836.25 & 1931.06 & 1931.06 & 1931.06 \\
$\mathrm{P}_{12}$ & $3 / 4 / 2 / 15$ & 1685.57 & 3147.47 & 1685.57 & 1685.57 & 1685.57 \\
$\mathrm{P}_{13}$ & $3 / 6 / 3 / 16$ & N/A & $>3600$ & 2763.52 & 2763.52 & 2763.52 \\
$\mathrm{P}_{14}$ & $3 / 7 / 3 / 18$ & N/A & $>3600$ & 3294.86 & 3292.13 & 3284.89 \\
$\mathrm{P}_{15}$ & $4 / 8 / 3 / 20$ & N/A & $>3600$ & 3493.84 & 3493.84 & 3493.84 \\
$\mathrm{P}_{16}$ & $4 / 8 / 3 / 22$ & N/A & $>3600$ & 3531.66 & 3496.86 & 3495.19 \\
\hline
\end{tabular}


Table 14. Results of $\overline{R P D}$ for the presented meta-heuristic algorithms on small-sized problems

\begin{tabular}{ccccc}
\hline Problem No. & Symbol & GOA $\overline{R P D}$ & IWO $\overline{R P D}$ & SA $\overline{R P D}$ \\
\hline $\mathrm{P}_{1}$ & $1 / 2 / 1 / 4$ & 0.00 & 0.00 & 0.00 \\
$\mathrm{P}_{2}$ & $2 / 2 / 1 / 5$ & 0.00 & 0.00 & 0.00 \\
$\mathrm{P}_{3}$ & $2 / 2 / 1 / 6$ & 0.14 & 0.00 & 0.14 \\
$\mathrm{P}_{4}$ & $2 / 2 / 1 / 7$ & 5.90 & 0.00 & 0.00 \\
$\mathrm{P}_{5}$ & $2 / 2 / 1 / 8$ & 0.00 & 0.00 & 0.00 \\
$\mathrm{P}_{6}$ & $2 / 2 / 1 / 9$ & 0.00 & 0.00 & 0.00 \\
$\mathrm{P}_{7}$ & $2 / 3 / 1 / 10$ & 0.21 & 0.00 & 0.21 \\
$\mathrm{P}_{8}$ & $2 / 3 / 2 / 11$ & 2.79 & 0.00 & 3.11 \\
$\mathrm{P}_{9}$ & $2 / 3 / 2 / 12$ & 0.00 & 0.00 & 0.00 \\
$\mathrm{P}_{10}$ & $2 / 4 / 2 / 13$ & 0.00 & 0.00 & 0.00 \\
$\mathrm{P}_{11}$ & $3 / 4 / 2 / 14$ & 0.00 & 0.00 & 0.00 \\
$\mathrm{P}_{12}$ & $3 / 4 / 2 / 15$ & 0.00 & 0.00 & 0.00 \\
$\mathrm{P}_{13}$ & $3 / 6 / 3 / 16$ & 1.24 & 0.00 & 0.82 \\
$\mathrm{P}_{14}$ & $3 / 7 / 3 / 18$ & 0.93 & 0.39 & 1.43 \\
$\mathrm{P}_{15}$ & $4 / 8 / 3 / 20$ & 0.42 & 0.20 & 0.20 \\
$\mathrm{P}_{16}$ & $4 / 8 / 3 / 22$ & 1.50 & 0.91 & 0.77 \\
\hline Mean & & 0.821 & 0.094 & 0.417
\end{tabular}

Table 15. Results of $\overline{R P D}$ for the presented meta-heuristic algorithms on medium and large-sized problems

\begin{tabular}{ccccc}
\hline Problem No. & Symbol & GOA $\overline{R P D}$ & IWO $\overline{R P D}$ & SA $\overline{R P D}$ \\
\hline $\mathrm{P}_{17}$ & $5 / 10 / 4 / 24$ & 0.00 & 0.03 & 0.00 \\
$\mathrm{P}_{18}$ & $5 / 11 / 4 / 26$ & 3.72 & 0.23 & 1.19 \\
$\mathrm{P}_{19}$ & $5 / 12 / 5 / 28$ & 2.78 & 1.06 & 3.72 \\
$\mathrm{P}_{20}$ & $6 / 13 / 5 / 30$ & 1.31 & 0.48 & 0.72 \\
$\mathrm{P}_{21}$ & $6 / 13 / 5 / 43$ & 2.93 & 1.34 & 2.38 \\
$\mathrm{P}_{22}$ & $6 / 13 / 6 / 46$ & 7.88 & 2.19 & 6.43 \\
$\mathrm{P}_{23}$ & $7 / 15 / 6 / 51$ & 8.15 & 3.04 & 8.35 \\
$\mathrm{P}_{24}$ & $7 / 16 / 6 / 52$ & 5.48 & 1.75 & 3.93 \\
$\mathrm{P}_{25}$ & $7 / 17 / 7 / 53$ & 3.49 & 0.84 & 2.99 \\
$\mathrm{P}_{26}$ & $8 / 19 / 7 / 55$ & 3.76 & 1.13 & 2.65 \\
$\mathrm{P}_{27}$ & $8 / 21 / 7 / 64$ & 4.20 & 1.45 & 2.79 \\
$\mathrm{P}_{28}$ & $8 / 21 / 8 / 66$ & 4.27 & 0.67 & 2.23 \\
$\mathrm{P}_{29}$ & $9 / 21 / 8 / 68$ & 7.94 & 2.62 & 6.28 \\
$\mathrm{P}_{30}$ & $9 / 22 / 8 / 69$ & 5.11 & 0.97 & 3.02 \\
$\mathrm{P}_{31}$ & $9 / 23 / 9 / 74$ & 6.28 & 1.24 & 4.08 \\
$\mathrm{P}_{32}$ & $9 / 24 / 9 / 74$ & 6.31 & 4.09 & 2.96 \\
\hline Mean & & 4.601 & 1.446 & 3.357 \\
\hline
\end{tabular}


Table 16. ANOVA test results for the presented meta-heuristic algorithms on small-sized problems

\begin{tabular}{cccccc}
\hline Source & DF & Adj SS & Adj MS & F-Value & P-Value \\
\hline Factor & 2 & 4.244 & 2.122 & 2.00 & 0.148 \\
Error & 45 & 47.852 & 1.063 & & \\
Total & 47 & 52.096 & & & \\
\hline
\end{tabular}

Table 17. ANOVA test results for the presented meta-heuristic algorithms on medium and large-sized problems

\begin{tabular}{cccccc}
\hline Source & DF & Adj SS & Adj MS & F-Value & P-Value \\
\hline Factor & 2 & 80.89 & 40.444 & 10.70 & 0.000 \\
Error & 45 & 170.10 & 3.780 & & \\
Total & 47 & 250.99 & & & \\
\hline
\end{tabular}

Table 18. Tukey test for comparison of the presented meta-heuristic algorithms at $95 \%$ confidence (small sizes)

\begin{tabular}{cccc}
\hline Factor & N & Mean & Grouping \\
\hline GOA & 16 & 0.821 & $A$ \\
SA & 16 & 0.417 & $A$ \\
IWO & 16 & 0.094 & $A$ \\
\hline
\end{tabular}

Table 19. Tukey test for comparison of the presented meta-heuristic algorithms at $95 \%$ confidence

\begin{tabular}{cccc}
\multicolumn{4}{c}{ (medium and large sizes) } \\
\hline Factor & $\mathbf{N}$ & Mean & Grouping \\
\hline GOA & 16 & 4.601 & $A$ \\
SA & 16 & 3.357 & $A$ \\
IWO & 16 & 1.446 & $B$ \\
\hline
\end{tabular}




\section{Figures:}

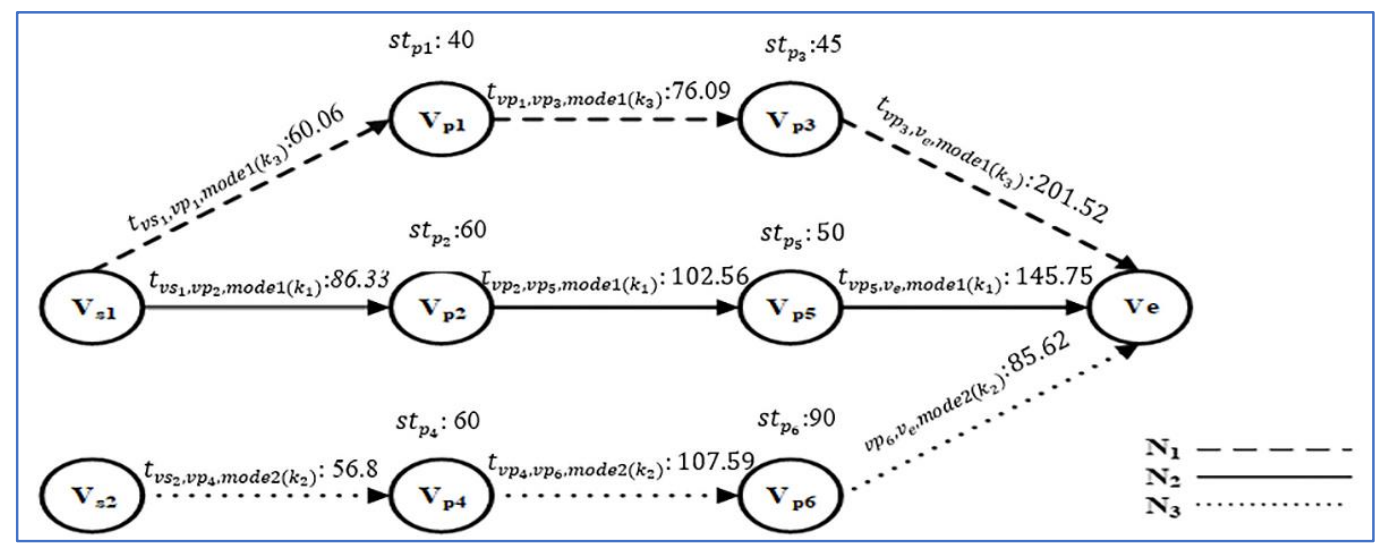

Figure 1. Directed graph of nurses' routes

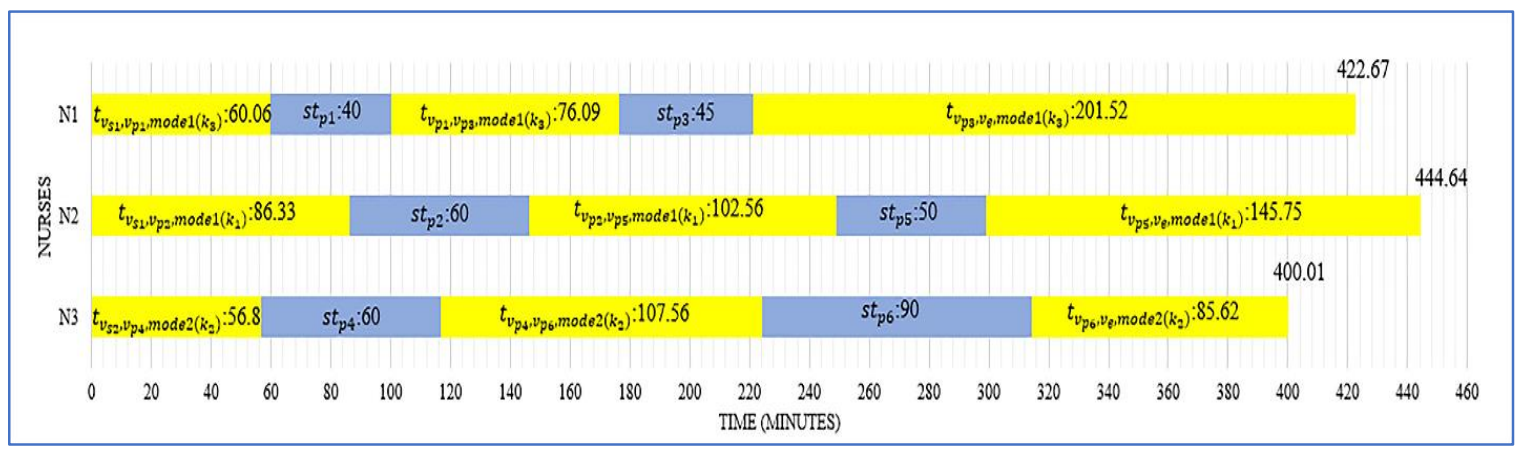

Figure 2. Gantt chart for nurses and patients

\begin{tabular}{|c|c|c|c|c|}
\hline \multirow{3}{*}{$\mathrm{N}_{1}$} & $\mathrm{Vs}_{1}$ & $\mathrm{Vs}_{2}$ & $\mathrm{Vp}_{1}$ & $\mathrm{Vp}_{3}$ \\
\hline & 0.682 & 0.571 & 0.671 & 0.864 \\
\hline & $\mathrm{Vs}_{1}$ & $\mathrm{Vs}_{2}$ & $\mathrm{Vp}_{2}$ & $\mathrm{Vp}_{5}$ \\
\hline \multirow[t]{2}{*}{$\mathrm{N}_{2}$} & 0.726 & 0.391 & 0.371 & 0.561 \\
\hline & $\mathrm{Vs}_{1}$ & $\mathrm{Vs}_{2}$ & $\mathrm{Vp}_{4}$ & $\mathrm{Vp}_{6}$ \\
\hline \multirow[t]{2}{*}{$\mathrm{N}_{3}$} & 0.127 & 0.491 & 0.321 & 0.571 \\
\hline & & & $\frac{N_{3}}{2}$ & \\
\hline
\end{tabular}

Figure 3. Representation of a solution 


\begin{tabular}{|ll|}
\hline$N_{1}$ & $V_{s 1} \rightarrow V_{p 1} \rightarrow V_{p 3} \rightarrow V_{e}$ \\
$N_{2}$ & $V_{s 1} \rightarrow V_{p 2} \rightarrow V_{p 5} \rightarrow V_{e}$ \\
$N_{3}$ & $V_{s 2} \rightarrow V_{p 4} \rightarrow V_{p 6} \rightarrow V_{e}$ \\
\hline
\end{tabular}

Figure 4. Nurses' routes related to Figure 3

\begin{tabular}{|c|c|c|c|c|c|c|c|}
\hline & $\mathrm{V}_{51}$ & $\mathrm{~V}_{52}$ & $\mathrm{~V}_{53}$ & $\mathrm{~V}_{\mathrm{pl}}$ & $\mathrm{V}_{\mathrm{p} 2}$ & $\mathrm{~V}_{\mathrm{p} 3}$ & \multirow[b]{3}{*}{$V_{p 7}$} \\
\hline \multirow[t]{2}{*}{$\mathrm{N}_{1}$} & 0.645 & 0.256 & 0.782 & 0.641 & 0.357 & 0.546 & \\
\hline & $\mathrm{V}_{\mathrm{s} 1}$ & $V_{52}$ & $V_{93}$ & $V_{p 4}$ & $\mathrm{~V}_{\mathrm{p} 5}$ & $\mathrm{~V}_{\mathrm{p} 6}$ & \\
\hline \multirow[t]{2}{*}{$\mathrm{N}_{2}$} & 0.865 & 0.358 & 0.156 & 0.685 & 0.875 & 0.347 & 0.715 \\
\hline & $\mathrm{V}_{\mathrm{sl}}$ & $\mathrm{V}_{32}$ & $\mathrm{~V}_{\mathrm{s3}}$ & $\mathrm{V}_{\mathrm{p} 8}$ & $\mathrm{~V}_{\mathrm{p} 9}$ & & \\
\hline \multirow[t]{2}{*}{$\mathrm{N}_{3}$} & 0.236 & 0.745 & 0.356 & 0.447 & 0.125 & & \\
\hline & $\mathrm{V}_{\mathrm{sl}}$ & $\mathrm{V}_{32}$ & $\mathrm{~V}_{\mathrm{s3}}$ & $\mathrm{V}_{\mathrm{p} 10}$ & $\mathrm{~V}_{\mathrm{p} 11}$ & $\mathrm{~V}_{\mathrm{p} 12}$ & \\
\hline \multirow[t]{3}{*}{$\mathrm{N}_{4}$} & 0.554 & 0.621 & 0.454 & 0.168 & 0.758 & 0.695 & \\
\hline & & $\mathrm{N}_{1}$ & $\mathrm{~N}_{2}$ & $\mathrm{~N}_{3}$ & $\mathrm{~N}_{4}$ & & \\
\hline & & 2 & 3 & 1 & 4 & & \\
\hline
\end{tabular}

Figure 5. Solution representation with the different length of strings

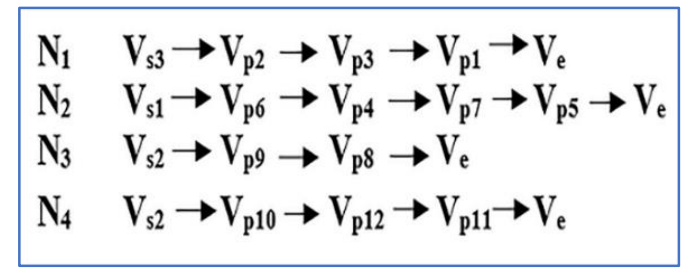

Figure 6. Nurses' routes related to Figure 5

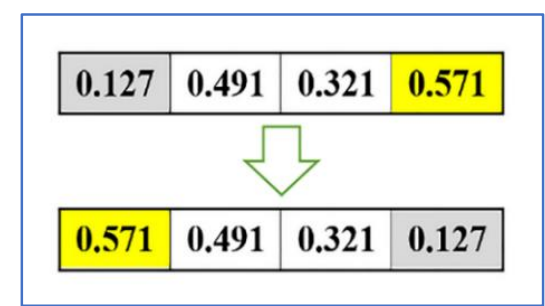

Figure 7. The procedure of Swap - Nurse string operator 


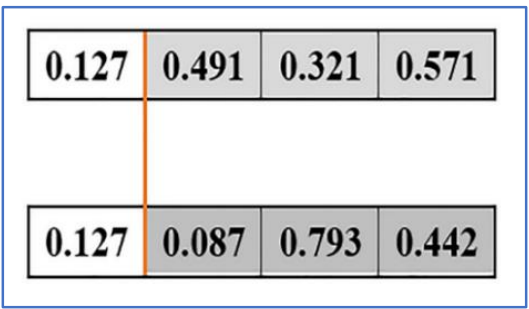

Figure 8. The procedure of Regenerating - Nurse string operator

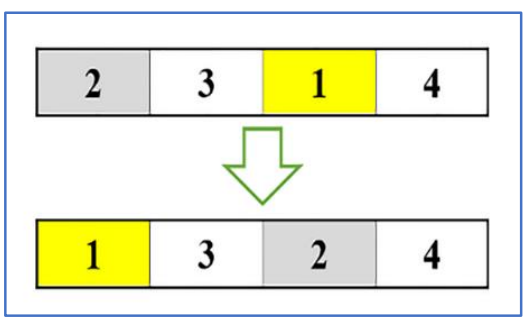

Figure 9. The procedure of Swap - Vehicle string operator

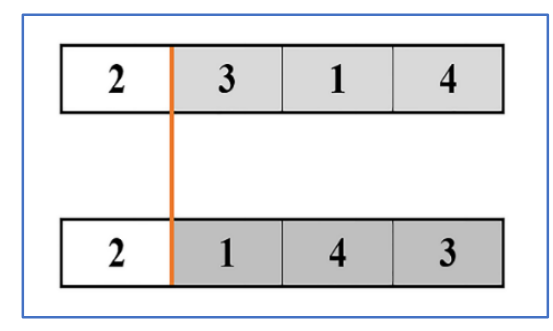

Figure 10. The procedure of Reordering - Vehicle string operator

\section{Initialization:}

Determine the values of $c_{\min }$ and $c_{\max }$;

Generate a random population of grasshoppers;

Calculate fitness for all grasshoppers and select the best grasshopper;

$T=$ the best solution;

While the stopping criterion is not met do

Update parameter $c$;

For each grasshopper

Normalize the distance between grasshoppers;

Update the position of each grasshopper;

Perform the neighborhood structures operators to change the position of each grasshopper in a random procedure;

Bring the current grasshopper back if it goes outside the boundaries;

\section{End for}

Update $T$ if there is a better solution;

End while

Figure 11. Steps of the presented Grasshopper Optimization Algorithm for the HHCRSP 


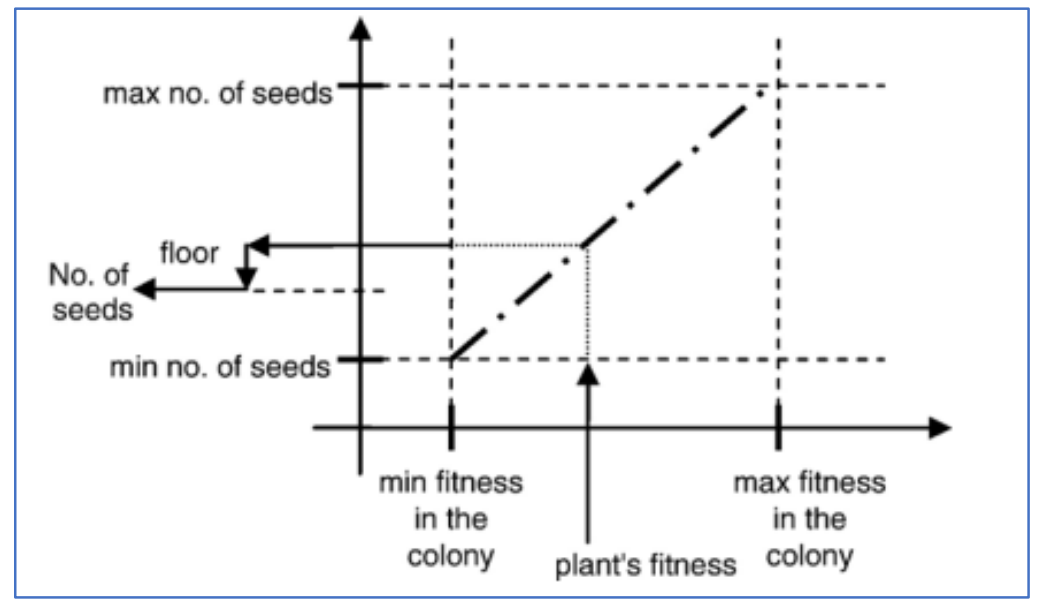

Figure 12. Procedure of producing seed [25]

\section{Initialization:}

Determine the values of the parameters of the algorithm;

Generate a random population of weeds $(W)$;

While the stop criterion is not met do

Compute the fitness of each weed $w$ in the population $W$;

Determine the best and the worst fitness in the population $W$;

Compute standard deviation for each iteration;

For each weed $w$ belongs to the population $W$

Compute the number of seeds that the plant $w$ should be produce, depending on its fitness;

Randomly distribute the generated seeds over the search space around the parent weed $w$;

Apply the neighborhood structures operators on the produced seeds in a random procedure;

Add the newly generated seeds to the population $W$;

End for

If the population size of $W$ is greater than the maximum limit of population size $\left(P_{\text {max }}\right)$

Apply elimination mechanism: Sort the population according to their fitness and eliminate the weeds with worse fitness to reach $P_{\max }$;

End if

End while

Figure 13. Steps of the presented Invasive Weed Optimization algorithm for the HHCRSP 


\section{Initialization:}

Generate an initial solution $\left(S_{0}\right)$;

Select an initial temperature $\left(T_{0}\right)$ and cooling schedule function;

$S=S_{0}$

$T=T_{0}$;

Repeat

\section{Repeat}

/n a fix temperature/

Produce a neighboring solution $S$ from $S$ by the neighborhood structure operators; Compute $\Delta E=f(\hat{S})-f(S)$;

If $\Delta E \leq 0$ then $S=S$; $\quad$ /Acceptance mechanism of neighboring solution/ Else accept $S$ with probability equal to $e^{\frac{-\Delta E}{T}}$;

\section{End If}

Until equilibrium condition /A number of iterations for each temperature/ $T=g(T)$; /Update temperature/

Update $S$ as the best solution;

Until stopping criteria is met;

Figure 14. Steps of the presented Simulated Annealing algorithm for the HHCRSP

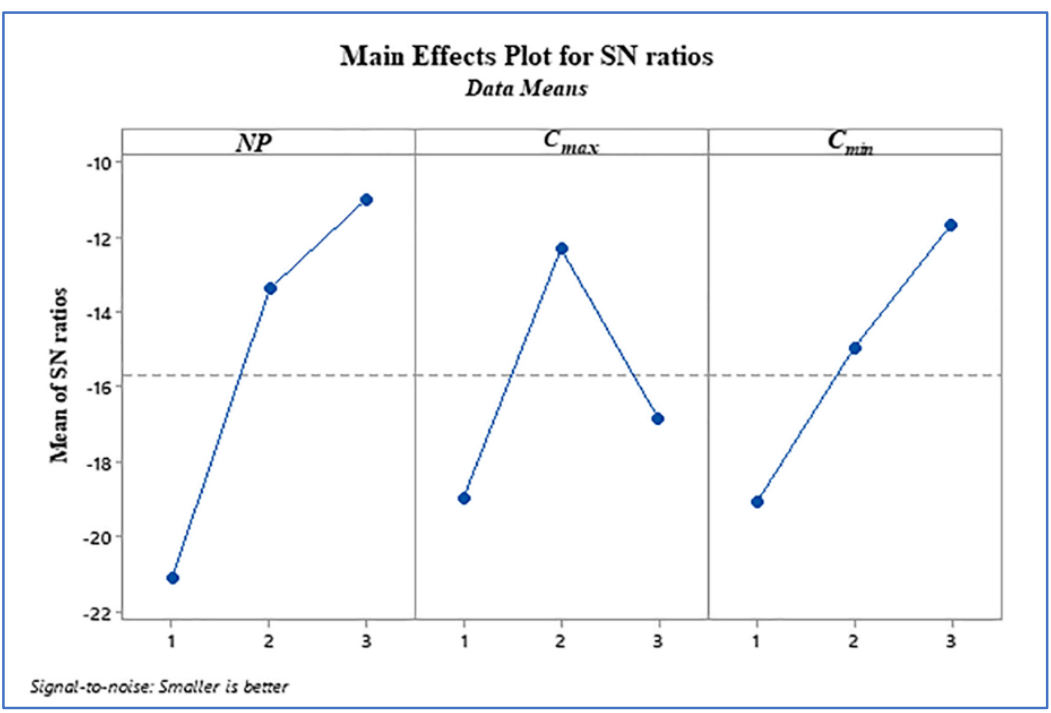

Figure 15. The average $\mathrm{S} / \mathrm{N}$ ratio plot at each level of the factors for objective function values in Grasshopper Optimization Algorithm 


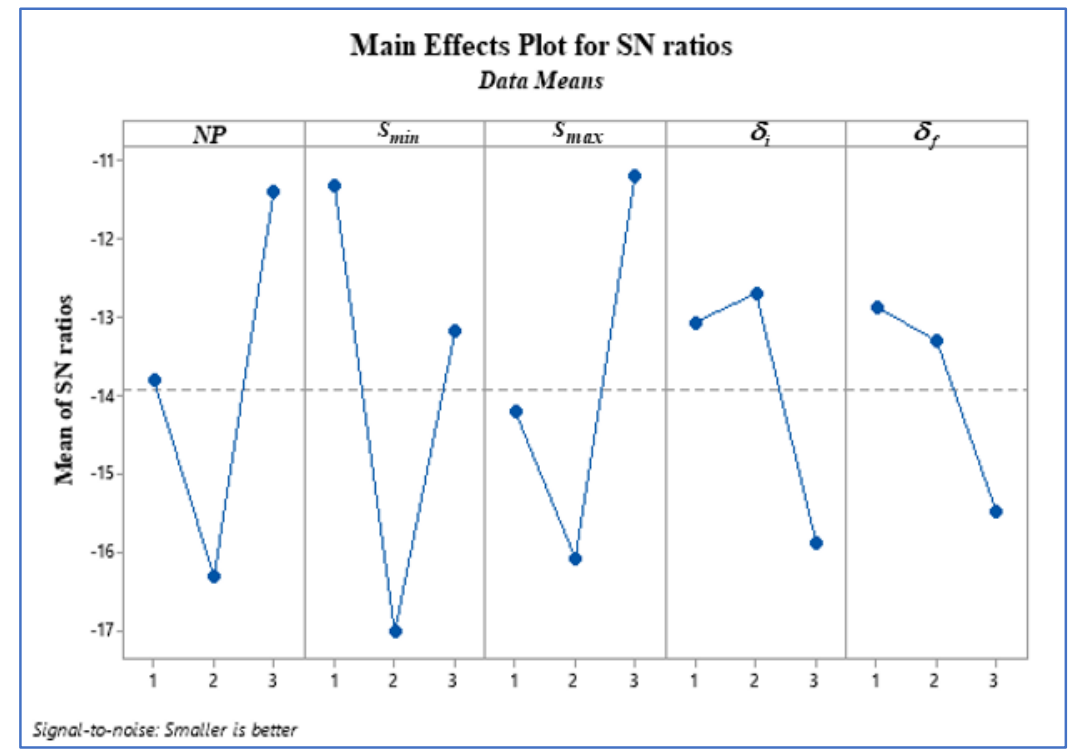

Figure 16. The average S/N ratio plot at each level of the factors for objective function values in Invasive Weed Optimization algorithm

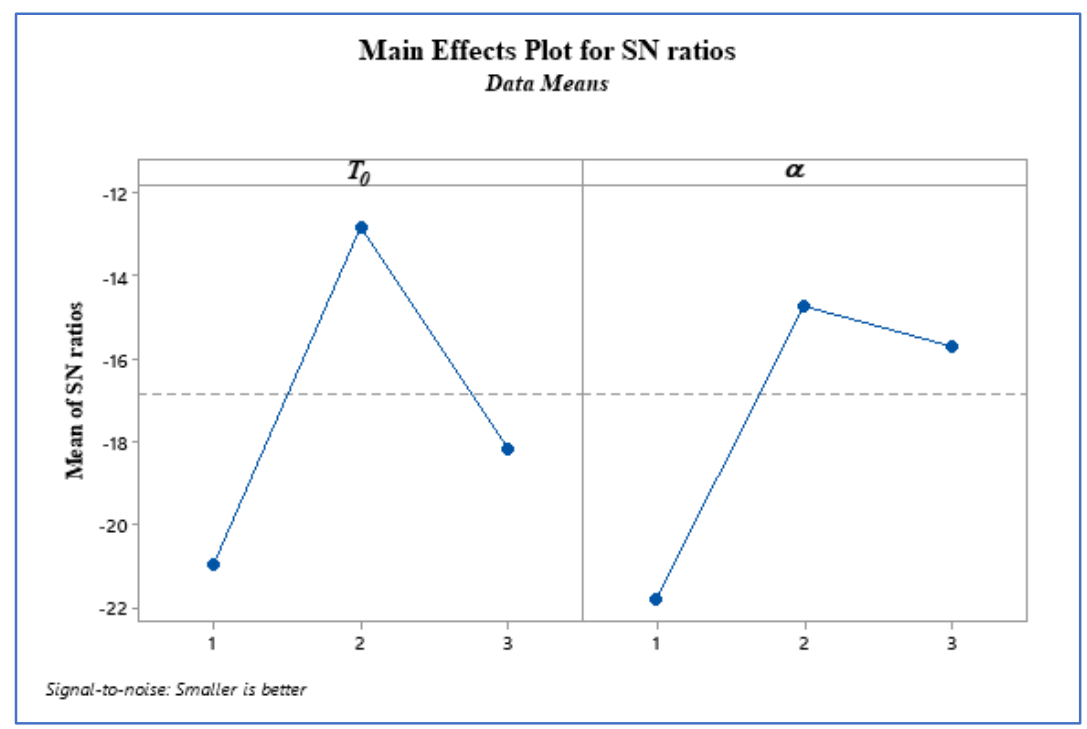

Figure 17. The average $\mathrm{S} / \mathrm{N}$ ratio plot at each level of the factors for objective function values in Simulated Annealing algorithm 


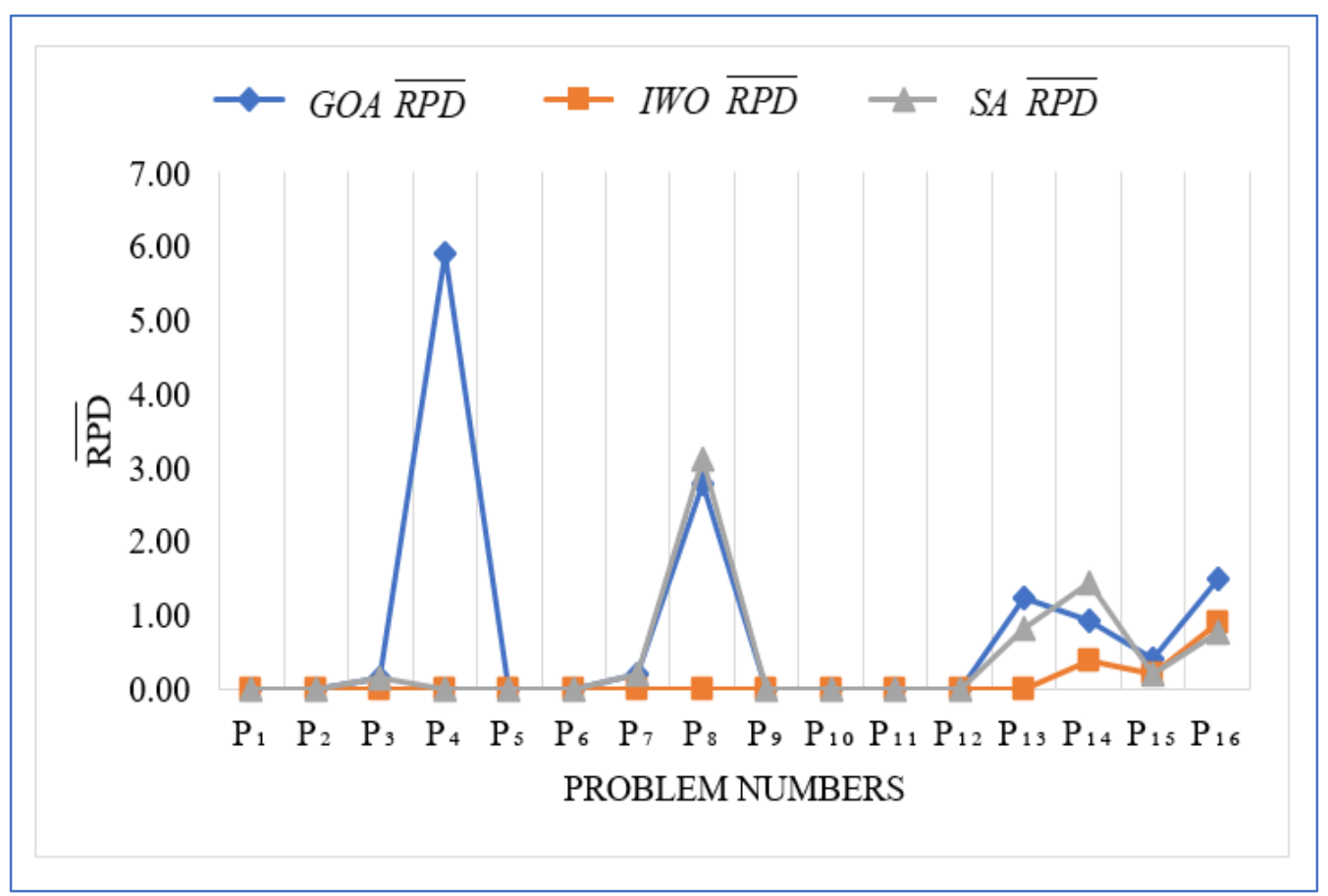

Figure 18. Results of the presented meta-heuristic algorithms on small-sized problems

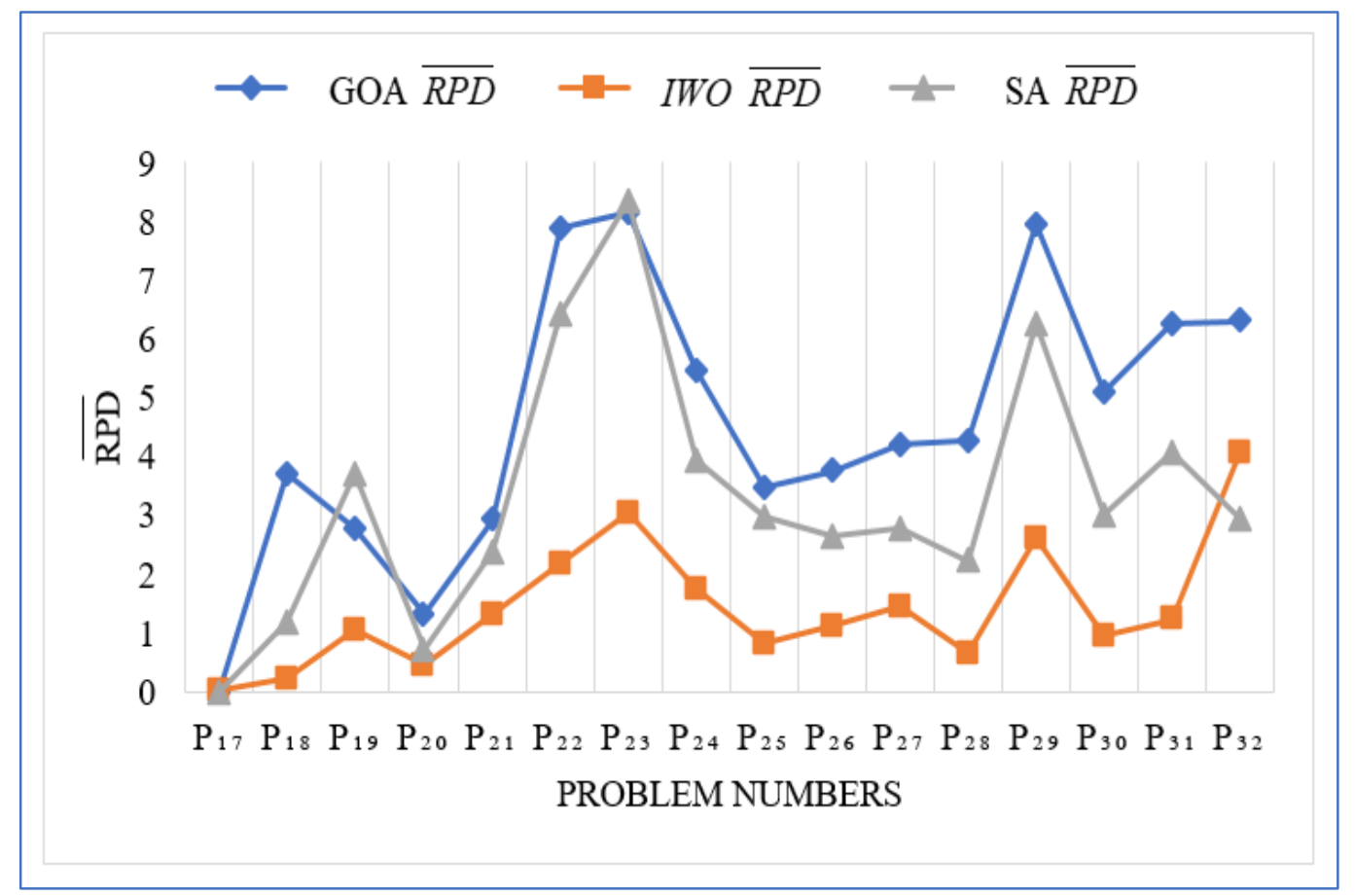

Figure 19. Results of the presented meta-heuristic algorithms on medium and large-sized problems 


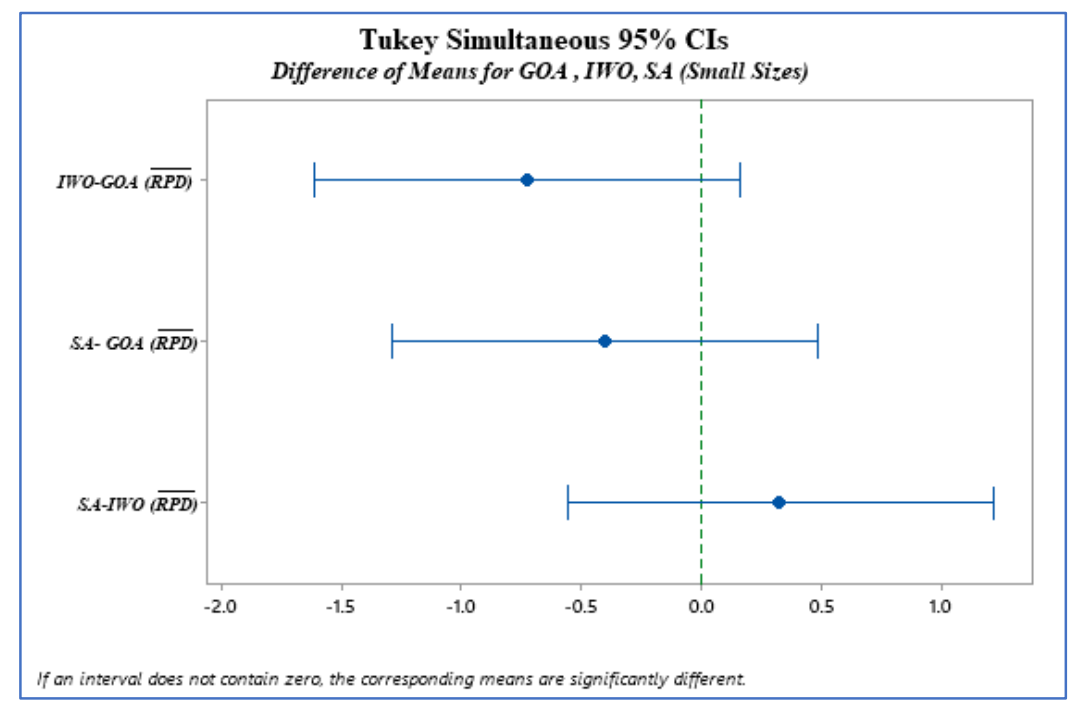

Figure 20. Tukey pairwise comparisons plot of the presented meta-heuristic algorithms (small sizes)

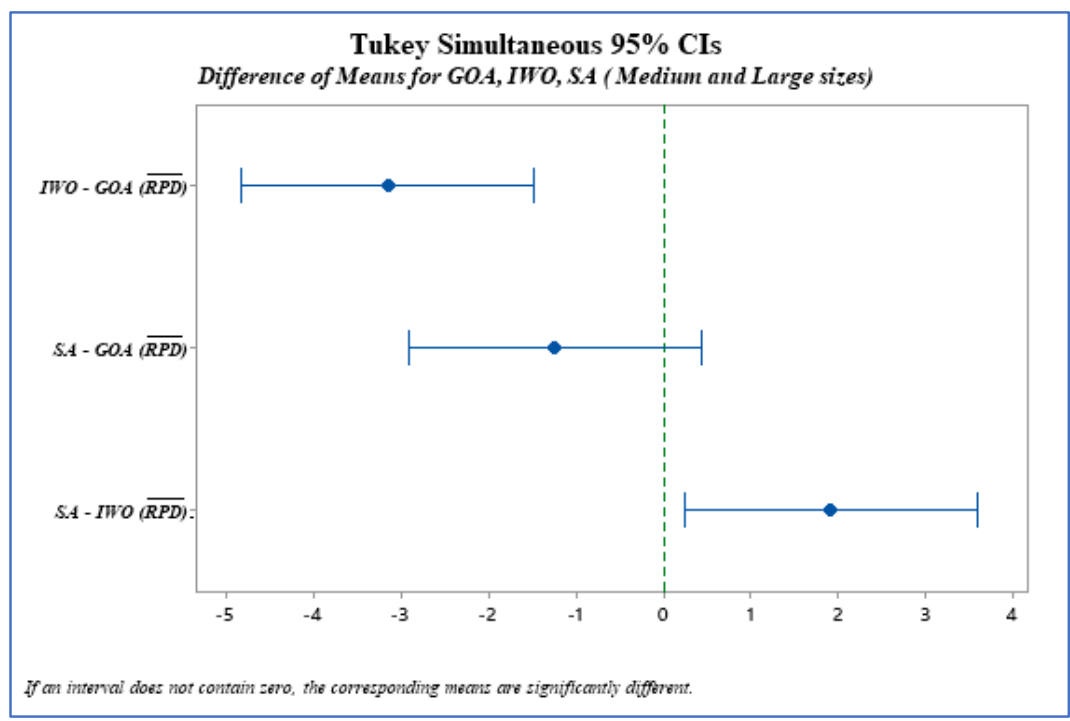

Figure 21. Tukey pairwise comparisons plot of the presented meta-heuristic algorithms (medium and large sizes) 


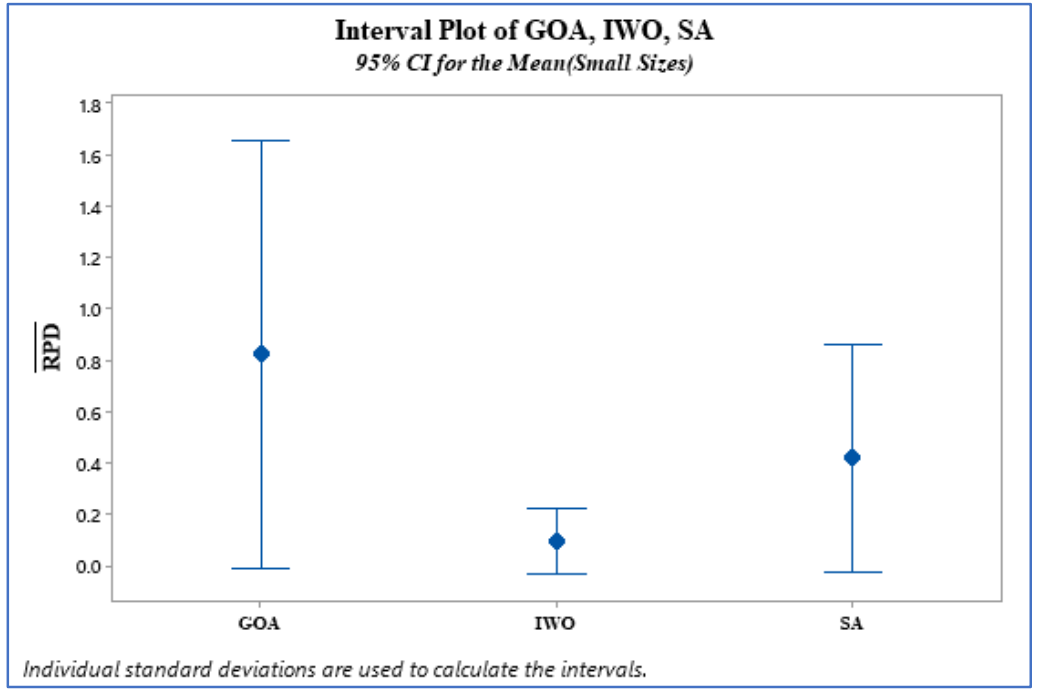

Figure 22. Mean interval plot of the presented meta-heuristic algorithms in terms of $\overline{R P D}$ measure (small sizes)

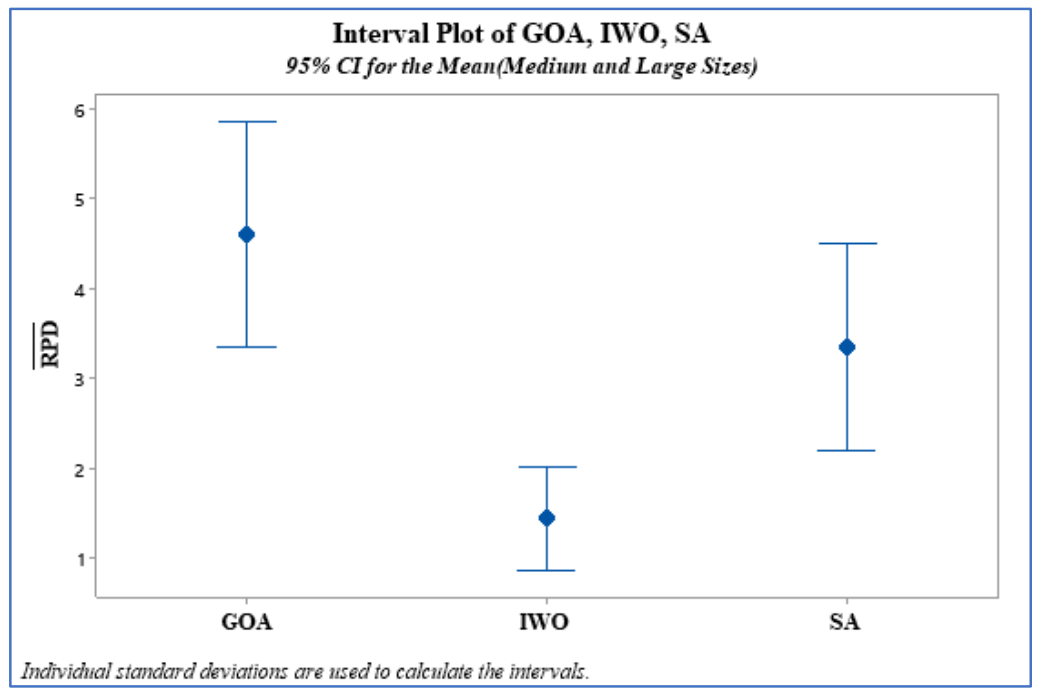

Figure 23. Mean interval plot of the presented meta-heuristic algorithms in terms of $\overline{R P D}$ measure (medium and large sizes) 